\title{
STOCHASTIC MODE REDUCTION FOR PARTICLE-BASED SIMULATION METHODS FOR COMPLEX MICROFLUID SYSTEMS*
}

\author{
PETER R. KRAMER ${ }^{\dagger}$ AND ANDREW J. MAJDA ${ }^{\ddagger}$
}

\begin{abstract}
We illustrate the stochastic mode reduction procedure as formulated recently by Majda, Timofeyev, and Vanden-Eijnden [Comm. Pure Appl. Math., 54 (2001), pp. 891-974] (MTV) on the equations of motion underlying various particle-based simulation approaches (such as Stokesian dynamics and Brownian dynamics) and the conceptually distinct dissipative particle dynamics (DPD) simulation approaches for complex microfluid systems. The resulting coarse-grained dynamics are compared and contrasted with each other. We show that the stochastic mode reduction procedure provides a way to recover the Smoluchowski dynamics for a standard model of multiple interacting particles in a fluid. The DPD, however, has some subtle aspects which obstruct the application of the stochastic mode reduction procedure. We discuss the mathematical and physical properties of the DPD method that underlie this difficulty.
\end{abstract}

Key words. Brownian dynamics, Brownian motion, dissipative particle dynamics, Smoluchowski reduction

AMS subject classifications. 60H10, 60H30, 60J60, 60J65, 60J70, 76R50, 82C31, 82C70, $82 \mathrm{C} 80$

DOI. $10.1137 /$ S0036139903422140

1. Introduction. In [24], we showed how the stochastic mode reduction framework of Majda, Timofeyev, and Vanden-Eijnden [31, 32, 33] (with MTV hereafter referring to [33]) could be used to assist in the design and analysis of the immersed boundary (IB) method [26, 25, 40] for numerically simulating microphysiological systems consisting of various elastic structures and particles immersed in a fluid with thermal fluctuations. The procedure exploited the smallness of the thermal Reynolds number, which implied a separation of time scales for the dynamics of the fluid and the immersed structures, and derived a simplified stochastic system for the immersed structures with the fluid variables eliminated using rigorous singular perturbation techniques $[14,18,29,39]$. It is natural to explore how this approach works on other numerical simulation techniques for complex fluid systems consisting of immersed structures and thermal fluctuations. We study here the application of the stochastic mode reduction framework of MTV to the equations underlying particle-based (PB) dynamics schemes such as Brownian dynamics [10], Stokesian dynamics [6, 46], and the conceptually distinct dissipative particle dynamics [1, 11, 13, 23, 35, 38]. These numerical approaches differ fundamentally from the IB method in that they do not treat the fluid dynamically, but rather immediately model its effective influence on the particles. The detailed equations of motion for each of the simulation methods involve some nontrivial approximations or assumptions, even before any numerical discretization is contemplated.

As we shall discuss briefly in the relevant sections, even though the equations

* Received by the editors January 30, 2002; accepted for publication (in revised form) June 11, 2003; published electronically December 31, 2003.

http://www.siam.org/journals/siap/64-2/42214.html

${ }^{\dagger}$ Department of Mathematical Sciences, Rensselaer Polytechnic Institute, 301 Amos Eaton Hall, 110 8th Street, Troy, NY 12180 (kramep@rpi.edu).

${ }^{\ddagger}$ Courant Institute of Mathematical Sciences, New York University, 251 Mercer Street, New York, NY 10012 (jonjon@cims.nyu.edu). This author's research was supported in part by ARO grant DAAD19-01-10810, NSF grant DMS9972865, and ONR grant N00014-96-1-0043. 
and variables underlying these simulation approaches are rather different from each other and the IB method, they each can be treated within the MTV framework to deduce a simplified system governing the immersed particles and structures when the viscous friction with the fluid is sufficiently strong. The notion of Reynolds number is a bit obscure for the methods discussed in the present paper, since viscosity does not enter as a governing parameter. Instead, we take the small parameter in the asymptotic analysis as a certain "thermal Kubo number," which is defined as the ratio of the rate of decorrelation of a particle's (Lagrangian) thermal velocity due to its advection into different fluid regions relative to its rate of decorrelation due to viscous damping. The limit of small thermal Kubo number seems appropriate for many microfluid applications and plays a universal role in preparing for a stochastic mode reduction procedure for the methods described in the present paper as well as the IB method discussed in [24].

For the IB method, a small thermal Kubo number implies a separation between the fast time scales of the fluid and the slow time scales of the immersed structures. For the methods treated in the present paper, the fluid is not treated dynamically. Instead, the system variables consist of the positions and momenta of the elementary components of the immersed structures. Stochastic forcing modeling thermal fluctuations is naturally applied to the momenta of the particles. A low thermal Kubo number implies that the time scale for the momentum variables is much faster than that of the position variables. The stochastic mode reduction procedure of MTV then suggests a simplification of the system through elimination of the momentum variables and the development of a new set of effective "coarse-grained" equations for the position variables. By coarse-grained dynamics for the particles in these simulation schemes we mean a simplified description involving only the particle positions and not the rapidly fluctuating velocities. The derivations of the simplified coarse-grained dynamics can be accomplished rigorously for the classical equations underlying PB methods such as Brownian dynamics and Stokesian dynamics, but the dissipative particle dynamics (DPD) scheme has some aspects which can obstruct the deduction of simplified effective dynamics.

The application of the MTV procedure to eliminate the fast momentum variables from the classical PB equations (section 2) yields the standard Smoluchowski (or Brownian dynamics) limit for the positions of the immersed structures, which have been computed previously with several other approaches $[10,37,47,49,50]$. The variation of the Chapman-Enskog procedure used in [47] has some foundational similarities to the method of derivation we present, but the present approach is based on a rigorous theorem [29], whereas the validity of the Chapman-Enskog expansion is less certain [45, 47]. Moreover, we calculate the differential operators associated with the simplified dynamics in a different way, involving a slight extension of the framework developed in MTV to a context in which the noise driving the fast modes is not diagonal.

A similar calculation can be made to derive effective equations for the elementary particles in a DPD system (section 3). These resulting equations look formally very similar to those arising from the classical PB equations. Due to the finite-ranged nature of the frictional interactions, however, degeneracies emerge which can spoil the MTV stochastic mode reduction procedure. The formal calculation appears to apply only for some fraction of realizations of the dynamics which increases with increasing density. We cannot yet, however, make this into a rigorous statement. It would be interesting to extend the rigorous aspect of the MTV framework to cover systems with degeneracies such as DPD, but we will not attempt this here. 
2. Application of stochastic mode reduction for PB simulation methods. Many scientists and engineers wishing to simulate microscopic fluid-particle systems with thermal fluctuations adopt a PB perspective in contrast to the fluidbased perspective of the IB method. In the PB setup, one appeals to the low Reynolds number of the system to eliminate the fluid degrees of freedom by assuming that the fluid is always in a quasi-steady state determined by the time-independent Stokes equations responding to whatever forces the particles happen to be exerting at that moment $[6,10,20]$. The fluid then ceases to be an independent dynamical quantity. The state of the system can be completely described by the positions $\mathbf{X}=\left\{\mathbf{X}_{\boldsymbol{\alpha}}\right\}_{\boldsymbol{\alpha} \in \mathcal{A}}$ and velocities $\mathbf{V}=\left\{\mathbf{V}_{\boldsymbol{\alpha}}\right\}_{\boldsymbol{\alpha} \in \mathcal{A}}$ of each immersed particle. These variables are updated according to Newton's laws, incorporating three kinds of forces:

(i) interparticle forces governed by some general potential of the particle configuration $\Phi(\mathbf{X})$,

(ii) hydrodynamic drag forces which are determined by the momentary positions and velocities of the particles, and

(iii) thermally fluctuating forces.

We provide details in section 2.1. Note that the approximation that the fluid evolves according to the quasi-steady Stokes equations requires stronger assumptions than simply noting that the Reynolds number is small [8], and these stronger assumptions are not necessarily met in practice [25].

While the PB simulation approaches are founded on these Newton law evolution equations for $\mathbf{X}$ and $\mathbf{V}$, these equations are not suitable for numerical simulation in many situations where the particle velocities decorrelate on a much faster time scale than the time over which the particle position variables change significantly. The equations of motion in this case are very stiff, but the disparity in time scales also permits a simplification of the system to one involving only the slow variables (particle positions). For the present example, this is known as the reduction of the full FokkerPlanck equation (involving position and momentum variables) to the Smoluchowski equation (involving only position variables). This procedure has been undertaken in various ways (formal asymptotic arguments [27], multiple time scale expansions [3], Chapman-Enskog expansion [47], projection operators [37, 49], asymptotic moment equation expansions [50], and asymptotic integration of stochastic differential equations $[10,22])$. We show here how this reduction can be performed within the MTV stochastic mode reduction framework. While the ideas behind the calculations are related to those in the earlier works (particularly the adaptation of the ChapmanEnskog expansion approach in [47] and the multiple time scale derivations for the case of a single particle in $[3,49]$ ), the MTV framework has a distinct organizational and computational structure which is systematic, fairly transparent, and founded on a rigorous theorem [29].

2.1. Formulation. We now write the equations on which the PB methods are based, with particle position and momentum resolved. For simplicity in exposition, we assume that all particles have the same mass $m$, though similar procedures can be applied to the more general case, as we shall discuss in section 2.5.

$$
\begin{aligned}
d \mathbf{X}_{\boldsymbol{\alpha}}(t)= & \mathbf{V}_{\boldsymbol{\alpha}}(t) d t \\
m d \mathbf{V}_{\boldsymbol{\alpha}}(t)=- & \sum_{\boldsymbol{\alpha}^{\prime} \in \mathcal{A}} \mathcal{R}_{\boldsymbol{\alpha}, \boldsymbol{\alpha}^{\prime}}(\mathbf{X}(t)) \mathbf{V}_{\boldsymbol{\alpha}^{\prime}}(t) d t-\nabla_{\boldsymbol{\alpha}} \Phi(\mathbf{X}(t)) d t \\
& \quad+\sqrt{2 k_{B} T} \sum_{\boldsymbol{\alpha}^{\prime} \in \mathcal{A}} \mathcal{S}_{\boldsymbol{\alpha}, \boldsymbol{\alpha}^{\prime}}(\mathbf{X}(t)) d \mathbf{W}_{\boldsymbol{\alpha}^{\prime}}
\end{aligned}
$$


These equations, as previously stated, are nothing more than Newton's second law, with the right-hand side of the second equation in (2.1) describing the three kinds of forces that are assumed to act on the particles. The first term is the model for the force felt by the particles due to the fluid, under the governing assumption that the fluid is always in a quasi-steady state responding to the currently applied forces and positions of the particles. The tensor $\mathcal{R}$ is the hydrodynamic resistance matrix $[6,20]$, which is symmetric, positive definite, and depends on the momentary particle configuration $\mathbf{X}$. Its entries $\mathcal{R}_{\boldsymbol{\alpha}, \boldsymbol{\alpha}^{\prime}}$ indicate how the motion of particle $\boldsymbol{\alpha}^{\prime}$ induces a hydrodynamic drag force on particle $\boldsymbol{\alpha}$. The second term in the second equation in (2.1) is just the force felt by a particle due to its direct interaction with other particles via the generalized potential $\Phi$, just as in the IB method, but it is now permissible for $\Phi$ to include external forces which do not conserve total momentum. The third term describes the random thermal fluctuations, with strength specified by the matrix $\mathcal{S}(t)$, which is (any) square root of the symmetric, positive definite matrix $\mathcal{R}(t)$ :

$$
\mathcal{R}(\mathbf{X})=\mathcal{S}(\mathbf{X}) \mathcal{S}^{\dagger}(\mathbf{X})
$$

The $\left\{\mathbf{W}_{\boldsymbol{\alpha}}\right\}_{\boldsymbol{\alpha} \in \mathcal{A}}$ are a collection of independent standard real-valued Brownian motions, which are mean zero Gaussian processes with stationary increments satisfying

$$
\left\langle d \mathbf{W}(t) \otimes d \mathbf{W}\left(t^{\prime}\right)\right\rangle=\mathcal{I} \delta\left(t-t^{\prime}\right) d t d t^{\prime} .
$$

Note that the Brownian motion $\mathbf{W}_{\boldsymbol{\alpha}}$ does not describe the Brownian motion of the particle with label $\boldsymbol{\alpha}$. Indeed, the Brownian motions of the particles are actually coupled, while $\left\{\mathbf{W}_{\boldsymbol{\alpha}}(t)\right\}_{\boldsymbol{\alpha} \in \mathcal{A}}$ are independent. One may think of the $\left\{\mathbf{W}_{\boldsymbol{\alpha}}(t)\right\}_{\boldsymbol{\alpha} \in \mathcal{A}}$ as a diagonalization of the thermal noise driving the particles. If the particles are well separated, then $\mathbf{W}_{\boldsymbol{\alpha}}(t)$ gives a leading order approximation to the thermal Brownian motion of particle $\boldsymbol{\alpha}$.

The equations of motion are supplemented by the initial conditions

$$
\mathbf{X}_{\boldsymbol{\alpha}}(t=0)=\mathbf{X}_{0, \boldsymbol{\alpha}}, \quad \mathbf{V}_{\boldsymbol{\alpha}}(t=0)=\mathbf{V}_{0, \boldsymbol{\alpha}} .
$$

In Stokesian dynamics $[6,46]$, an imposed background spatially linear flow $\mathbf{u}^{\infty}(\mathbf{x})$ is admitted, and can be incorporated into our framework by generalizing the resistance force term in (2.1) to

$$
-\sum_{\boldsymbol{\alpha}^{\prime} \in \mathcal{A}} \mathcal{R}_{\boldsymbol{\alpha}, \boldsymbol{\alpha}^{\prime}}(\mathbf{X}(t)) \cdot\left(\mathbf{V}_{\boldsymbol{\alpha}^{\prime}}(t)-\mathbf{u}^{\infty}\left(\mathbf{X}_{\boldsymbol{\alpha}^{\prime}}(t)\right)\right)+\left(\mathcal{R}^{E}(\mathbf{X}(t)): \mathcal{E}^{\infty}\right)_{\boldsymbol{\alpha}}
$$

Here $\mathcal{R}^{E}(\mathbf{X})$ is another resistance tensor (with one particle and two spatial indices) relating to the imposed (constant) strain $\mathcal{E}^{\infty}=\frac{1}{2}\left(\nabla \mathbf{u}^{\infty}+\left(\nabla \mathbf{u}^{\infty}\right)^{\dagger}\right)$. We see that this generalization is mathematically equivalent to adding a (nonconservative) force

$$
-\sum_{\boldsymbol{\alpha}^{\prime} \in \mathcal{A}} \mathcal{R}_{\boldsymbol{\alpha}, \boldsymbol{\alpha}^{\prime}}(\mathbf{X}(t)) \cdot \mathbf{u}^{\infty}\left(\mathbf{X}_{\boldsymbol{\alpha}^{\prime}}(t)\right)+\left(\mathcal{R}^{E}(\mathbf{X}(t)) \mathcal{E}^{\infty}\right)_{\boldsymbol{\alpha}}
$$

which can be handled in the same way as the interparticle force term. To avoid extra burden in the calculations and nondimensionalization, we therefore proceed with no imposed flow $\mathbf{u}^{\infty} \equiv 0$ but comment in subsection 2.3 on how it could be incorporated.

\subsection{Nondimensionalization.}

2.2.1. Parameters of externally specified functions. We nondimensionalize the potential for the interacting particles by

$$
\Phi(\mathbf{X})=F \ell_{f} \tilde{\Phi}\left(\mathbf{X} / \ell_{f}\right)
$$


where $F$ is a typical force amplitude and $\ell_{f}$ is a length scale characterizing the range of the interparticle forces. Note that we are now using a force magnitude rather than a force density magnitude as we did for the IB method in [24]; this is due to the shift from a fluid-based to $\mathrm{PB}$ perspective. We let $\ell_{0}$ be a length scale characterizing the typical initial separation between particles, and $V_{0}$ a typical magnitude of the initial particle velocities, and write

$$
\mathbf{X}_{0, \boldsymbol{\alpha}}=\ell_{0} \tilde{\mathbf{X}}_{0, \boldsymbol{\alpha}}, \quad \mathbf{V}_{0, \boldsymbol{\alpha}}=V_{0} \tilde{\mathbf{V}}_{0, \boldsymbol{\alpha}}
$$

where $\tilde{\mathbf{X}}_{0, \boldsymbol{\alpha}}$ and $\tilde{\mathbf{V}}_{0, \boldsymbol{\alpha}}$ are nondimensionalized initial data which are to be thought of (at least formally) as order unity.

The resistance tensor $\mathcal{R}$ depends both on the current configuration $\mathbf{X}(t)$ of the particles and on their sizes and shapes. For now, we will assume that all particles are identical, with maximal diameter $a$ and hydrodynamic drag friction $\gamma$. In the dilute limit (where the particle separation scale is much larger than the particle size $a$ ), we would have $\mathcal{R}=\gamma \mathcal{I}$, where $\mathcal{I}$ is the identity matrix, but the resistance tensor in a nondilute solution will have both diagonal and off-diagonal modifications due to hydrodynamic coupling between different particles. We can express the resistance tensor in terms of a nondimensional function $\tilde{\mathcal{R}}$ (depending on the shape of the particles) [20] which has order unity variations (i.e., is properly normalized):

$$
\mathcal{R}(\mathbf{X})=\gamma \tilde{\mathcal{R}}(\mathbf{X} / a)
$$

The amplitude of the thermal forces is similarly expressed in terms of a nondimensional normalized function:

$$
\mathcal{S}(\mathbf{X})=\sqrt{\gamma} \tilde{\mathcal{S}}(\mathbf{X} / a)
$$

with $\tilde{\mathcal{S}} \tilde{\mathcal{S}}^{\dagger}=\tilde{\mathcal{R}}$. We will assume that $\mathcal{R}$ is appropriately mollified to avoid singularities as two or more particles coalesce, as must be done in any numerical implementation.

2.2.2. Reference units. We use the following:

(i) length scale $\ell_{T}=a$,

(ii) time scale $\tau_{T}=\sqrt{m a^{2} / k_{B} T}$,

(iii) mass scale $m_{T}=m$.

These units are chosen with motivation similar to those used to nondimensionalize the IB equations in [24]. We have simply chosen $\ell_{T}=a$, both because physiological systems are typically composed of extended structures such as polymers and membranes which will be modeled by stringing together particles separated by distances comparable to their sizes and because it matches most closely the nondimensionalization choices made for other simulation methods discussed in this paper. The characteristic velocity is again the order of magnitude of the thermal velocity of a particle:

$$
V_{T}=\frac{\ell_{T}}{\tau_{T}}=\sqrt{\frac{k_{B} T}{m}}
$$

\subsubsection{Nondimensional groups.}

(i) The thermal Kubo number

$$
\mathrm{Ku}_{\mathrm{T}}=\frac{\sqrt{m k_{B} T}}{\gamma a},
$$


which describes the ratio of the time scale of frictional decorrelation $m / \gamma$ to the time scale $\ell_{T} / V_{T}$ over which the particles would move across a spatial structural length $\ell_{T}=a$. Note that if the fluid density $\rho$ is comparable to the particle mass density $m / a^{3}$, we can also think of the thermal Kubo number as a thermal particle Reynolds number $\ell_{T} V_{T} / \nu$, because the self-friction constant scales as $\gamma \sim \rho \nu a$. We note, though, that the equations for $\mathrm{PB}$ dynamics are most accurate in the limit in which the particle mass density is much greater than that of the fluid [8, 21, 30, 36, 44, 48].

(ii) Nondimensionalized measures of the effects of structural forces and initial velocity,

$$
\phi=\frac{F a}{k_{B} T}, \quad \Upsilon=\frac{V_{0}}{V_{T}} .
$$

(iii) The length scale ratios

$$
\tilde{\ell}_{f}=\frac{\ell_{f}}{a}, \quad \tilde{\ell}_{0}=\frac{\ell_{0}}{a} .
$$

2.2.4. Nondimensionalized PB dynamics. Nondimensionalizing with respect to the reference units described in section 2.2.2 and denoting nondimensional variables (but not externally prescribed functions) by the same notation as for their dimensional form, we obtain

$$
\begin{aligned}
d \mathbf{X}_{\boldsymbol{\alpha}}(t)= & \mathbf{V}_{\boldsymbol{\alpha}}(t) d t \\
d \mathbf{V}_{\boldsymbol{\alpha}}(t)=- & \phi \nabla_{\boldsymbol{\alpha}} \tilde{\Phi}\left(\mathbf{X}(t) / \tilde{\ell}_{f}\right) d t-\sum_{\boldsymbol{\alpha}^{\prime} \in \mathcal{A}} \mathrm{Ku}_{\mathrm{T}}^{-1} \tilde{\mathcal{R}}_{\boldsymbol{\alpha}, \boldsymbol{\alpha}^{\prime}}(\mathbf{X}(t)) \mathbf{V}_{\boldsymbol{\alpha}^{\prime}}(t) d t \\
& \quad+\sqrt{2} \mathrm{Ku}_{\mathrm{T}}^{-1 / 2} \tilde{\mathcal{S}}_{\boldsymbol{\alpha}, \boldsymbol{\alpha}^{\prime}}(\mathbf{X}(t)) d \mathbf{W}_{\boldsymbol{\alpha}^{\prime}} .
\end{aligned}
$$

The nondimensionalized initial conditions are

$$
\mathbf{X}_{\boldsymbol{\alpha}}(t=0)=\tilde{\ell}_{0} \tilde{\mathbf{X}}_{0, \boldsymbol{\alpha}}, \quad \mathbf{V}_{\boldsymbol{\alpha}}(t=0)=\Upsilon \tilde{\mathbf{V}}_{0, \boldsymbol{\alpha}} .
$$

2.3. Effective dynamics for PB dynamics at low thermal Kubo number. Elimination of the fast momentum modes yields the following description of the evolution of the positions of the particles.

Proposition 2.1 ( $\mathrm{PB}$ dynamics at small Kubo number). In the limit $\mathrm{Ku}_{\mathrm{T}} \rightarrow 0$ with all other nondimensional quantities held fixed, the solution for the particle positions $\left\{\mathbf{X}_{\boldsymbol{\alpha}}(t)\right\}_{\boldsymbol{\alpha}}$ obtained from the complete system (2.1) and rescaled in time as

$$
\overline{\mathbf{X}}_{\boldsymbol{\alpha}}(t)=\lim _{\mathrm{Ku}_{\mathrm{T}} \rightarrow 0} \mathbf{X}_{\boldsymbol{\alpha}}\left(t / \mathrm{Ku}_{\mathrm{T}}\right)
$$

converges in law to the solution of the following simplified closed stochastic differential system involving only the particle positions $\left\{\overline{\mathbf{X}}_{\boldsymbol{\alpha}}(t)\right\}$ :

$$
\begin{aligned}
d \overline{\mathbf{X}}_{\boldsymbol{\alpha}}(t) & =\overline{\mathbf{V}}_{\boldsymbol{\alpha}}(\overline{\mathbf{X}}(t)) d t+\sum_{\boldsymbol{\alpha}^{\prime} \in \mathcal{A}} \mathcal{S}_{\boldsymbol{\alpha} \boldsymbol{\alpha}^{\prime}}(\overline{\mathbf{X}}(t)) d \mathbf{W}_{\boldsymbol{\alpha}^{\prime}}(t), \\
\overline{\mathbf{X}}_{\boldsymbol{\alpha}}(t=0) & =\tilde{\mathbf{X}}_{0, \boldsymbol{\alpha}},
\end{aligned}
$$

where the stochastic real white noise terms $d \mathbf{W}_{\boldsymbol{\alpha}}(t)$ are defined near (2.2) and are given the Itô interpretation. The explicit expression for the drift term is

$$
\overline{\mathbf{V}}_{\boldsymbol{\alpha}}(\mathbf{X})=\sum_{\boldsymbol{\alpha}^{\prime} \in \mathcal{A}}\left[-\left((\tilde{\mathcal{R}}(\mathbf{X}))^{-1}\right)_{\boldsymbol{\alpha}, \boldsymbol{\alpha}^{\prime}} \cdot \nabla_{\boldsymbol{\alpha}^{\prime}} \tilde{\Phi}\left(\mathbf{X} / \tilde{\ell}_{f}\right)+\nabla_{\boldsymbol{\alpha}^{\prime}} \cdot\left(\tilde{\mathcal{R}}^{-1}(\mathbf{X})\right)_{\boldsymbol{\alpha}^{\prime}, \boldsymbol{\alpha}}\right]
$$


and the matrix coefficients of the stochastic terms are

$$
\mathcal{S}_{\boldsymbol{\alpha} \boldsymbol{\alpha}^{\prime}}(\mathbf{X})=\sqrt{2}\left(\tilde{\mathcal{R}}^{-1 / 2}(\mathbf{X})\right)_{\boldsymbol{\alpha}, \boldsymbol{\alpha}^{\prime}} .
$$

The drift term here includes not only the motion of the particles responding to the interparticle forces under the quasi-steady assumption for the fluid state, but a "divergence-drift" term, which appears due to the spatial dependence of $\tilde{\mathcal{R}}$ and is present even in the absence of forces on the particles [10,19, 41]. The clearest way to understand the divergence-drift is by noting that the diffusion correlation tensor

$\mathcal{D}(\mathbf{r}) \equiv \frac{1}{2} \frac{d}{d t}\left\langle\left(\mathbf{X}_{\boldsymbol{\alpha}}(t)-\mathbf{X}_{\boldsymbol{\alpha}}\left(t^{\prime}\right)\right) \otimes\left(\mathbf{X}_{\boldsymbol{\alpha}^{\prime}}(t)-\mathbf{X}_{\boldsymbol{\alpha}^{\prime}}\left(t^{\prime}\right)\right) \mid \mathbf{X}_{\boldsymbol{\alpha}}\left(t^{\prime}\right)=\mathbf{x}+\mathbf{r}, \mathbf{X}_{\boldsymbol{\alpha}^{\prime}}\left(t^{\prime}\right)=\mathbf{x}\right\rangle_{t=t^{\prime}}$

is, for the nondimensionalized PB system, exactly $\mathcal{D}(\mathbf{X})=(\tilde{\mathcal{R}}(\mathbf{X}))^{-1}$, so that the divergence-drift term is $\nabla \cdot \mathcal{D}$. Roughly speaking, the divergence-drift arises because variable diffusivity induces a bias to the mean particle motion due to the asymmetric strength of fluctuations in opposite directions. This term is important only when two particles happen to be close together relative to their sizes [15, pp. 232-233], and is omitted in simulations based only on Oseen or Rotne-Prager approximations to the resistance tensor $[2,9,10,17,28,43]$.

The coarse-grained PB dynamics respect the Einstein relation between mobility and diffusion correlations. Unlike the IB method, it is permissible for the potential in the PB dynamics to include external forces (so that total momentum need not be conserved), and the conclusions of Proposition 2.1 are unchanged.

If a steady background flow $\mathbf{u}^{\infty}(\mathbf{x})$ is imposed which is not too strong relative to the thermal fluctuations, the only change in the effective dynamics is the addition of the nondimensionalized form of the terms $\mathbf{u}^{\infty}\left(\mathbf{X}_{\boldsymbol{\alpha}}(t)\right)+\left(\mathcal{R}^{-1} \cdot \mathcal{R}^{E}: \mathcal{E}^{\infty}\right)_{\boldsymbol{\alpha}}$ to the right-hand side of the expression (2.4) for the effective drift, in agreement with how these effects are handled within Stokesian dynamics [6].

2.4. Stochastic mode reduction for PB dynamics. We now derive Proposition 2.1, following the general prescription from MTV, which we presented in some detail in [24]. We focus here only on those aspects of the calculation that are particular to the PB equations; the basic notation and formalism are the same as in [24].

2.4.1. Kolmogorov backward equation formalism. We identify $\varepsilon=\mathrm{Ku}_{\mathrm{T}}$ as the small parameter and rescale to a longer time $t \rightarrow t / \varepsilon$. We denote the system variables in terms of the collection of particle positions $\mathbf{X}$ and velocities $\mathbf{V}$. Then the Kolmogorov backward equation under this time rescaling can be written as

$$
\begin{aligned}
& -\frac{\partial \rho^{\varepsilon}(s, \mathbf{X}, \mathbf{V} \mid t)}{\partial s}=\varepsilon^{-2} \mathcal{L}_{1} \rho^{\varepsilon}+\varepsilon^{-1} \mathcal{L}_{2} \rho^{\varepsilon}, \\
& \rho^{\varepsilon}(s=t, \mathbf{X}, \mathbf{V} \mid t)=f(\mathbf{X}, \mathbf{V}),
\end{aligned}
$$

with differential operators

$$
\begin{aligned}
& \mathcal{L}_{1}=\sum_{\boldsymbol{\alpha}, \boldsymbol{\alpha}^{\prime} \in \mathcal{A}}-\mathbf{V}_{\boldsymbol{\alpha}} \cdot \tilde{\mathcal{R}}_{\boldsymbol{\alpha}, \boldsymbol{\alpha}^{\prime}}(\mathbf{X}) \cdot \frac{\partial}{\partial \mathbf{V}_{\boldsymbol{\alpha}^{\prime}}}+\frac{\partial}{\partial \mathbf{V}_{\boldsymbol{\alpha}}} \cdot \tilde{\mathcal{R}}_{\boldsymbol{\alpha}, \boldsymbol{\alpha}^{\prime}}(\mathbf{X}) \cdot \frac{\partial}{\partial \mathbf{V}_{\boldsymbol{\alpha}^{\prime}}}, \\
& \mathcal{L}_{2}=-\sum_{\boldsymbol{\alpha} \in \mathcal{A}} \phi \nabla_{\boldsymbol{\alpha}} \tilde{\Phi}\left(\frac{\mathbf{X}(t)}{\tilde{\ell}_{f}}\right) \cdot \frac{\partial}{\partial \mathbf{V}_{\boldsymbol{\alpha}}}+\mathbf{V}_{\boldsymbol{\alpha}} \cdot \frac{\partial}{\partial \mathbf{X}_{\boldsymbol{\alpha}}}
\end{aligned}
$$


2.4.2. Asymptotic expansion of solution. We assume that $f$ depends only on the slow variables $f=f(\mathbf{X})$ to avoid consideration of irrelevant initial transients. The solvability conditions for the equations in the asymptotic hierarchy are obtained by integrating against the invariant measure $\pi_{O U}$ associated with the operator $\mathcal{L}_{1}$, which here is

$$
\pi_{O U}(\mathbf{V})=\left(\prod_{\boldsymbol{\alpha} \in \mathcal{A}} \frac{1}{2 \pi}\right) \exp \left(-\frac{1}{2} \sum_{\boldsymbol{\alpha} \in \mathcal{A}}\left|\mathbf{V}_{\boldsymbol{\alpha}}\right|^{2}\right) .
$$

The first two equations in the asymptotic hierarchy are solved in the same way as in [24], and the solvability condition resulting from the third equation is again

$$
\begin{aligned}
-\frac{\partial \rho_{0}}{\partial s} & =-\mathbf{E}_{O U} \mathcal{L}_{2} \mathcal{L}_{1}^{-1} \mathcal{L}_{2} \mathbf{E}_{O U} \rho_{0}, \\
\rho_{0}(s=t, \mathbf{X} \mid t) & =f(\mathbf{X})
\end{aligned}
$$

thus the main task is to compute explicitly

$$
\overline{\mathcal{L}} \equiv-\mathbf{E}_{O U} \mathcal{L}_{2} \mathcal{L}_{1}^{-1} \mathcal{L}_{2} \mathbf{E}_{O U}
$$

2.4.3. Explicit computation of limiting PDE. Thus far, our derivation largely coincides with the expansion procedure in [47]. However, instead of diagonalizing $\mathcal{L}_{1}$ in terms of Hermite polynomials, we follow in the spirit of the procedure developed in Appendix B of MTV. We show how this approach can be modified to directly treat a case such as the present one, where $\mathcal{L}_{1}$ is not diagonal in the fast variables $\mathbf{V}$.

We pass again to fast Fourier variables $\mathbf{P}=\left\{\mathbf{p}_{\boldsymbol{\alpha}}\right\}$ defined through

$$
\hat{g}(\mathbf{P})=\int_{\mathbb{R}^{N}} \exp \left[i \sum_{\boldsymbol{\alpha} \in \mathcal{A}} \mathbf{p}_{\boldsymbol{\alpha}} \cdot \mathbf{V}_{\boldsymbol{\alpha}}\right] g(\mathbf{V}) d \mathbf{V} .
$$

The differential operators act on functions of $\mathbf{P}$ and $\mathbf{X}$ as follows:

$$
\begin{aligned}
& \hat{\mathcal{L}}_{1}=\frac{\partial}{\partial \mathbf{P}} \cdot \tilde{\mathcal{R}}(\mathbf{X}) \cdot \mathbf{P}-\mathbf{P} \cdot \tilde{\mathcal{R}}(\mathbf{X}) \cdot \mathbf{P}, \\
& \hat{\mathcal{L}}_{2}=i \phi \mathbf{P} \cdot \nabla_{\mathbf{X}} \tilde{\Phi}\left(\frac{\mathbf{X}}{\tilde{\ell}_{f}}\right)-i \frac{\partial}{\partial \mathbf{P}} \cdot \frac{\partial}{\partial \mathbf{X}} .
\end{aligned}
$$

We have written these operators in a "grand matrix" form, which will facilitate later computations. We just think of quantities like $\mathbf{X}$ and $\mathbf{P}$ as vectors with $3 N$ components, and matrices like $\tilde{\mathcal{R}}$ as $3 N \times 3 N$ matrices. Scalar products between vectors (and vector operators) then involve summing over particle labels in addition to the Cartesian dimensions.

We now need to compute $\hat{\mathcal{L}}_{1}^{-1}$ and will have to generalize Lemma A.2 in MTV to allow for the nondiagonal structure of $\hat{\mathcal{L}}_{1}$. Since a similar result will be needed for the DPD calculation below, we present here a lemma sufficient for both calculations.

Lemma 2.2. Suppose we are given a first order differential operator of the form

$$
\hat{\mathcal{L}}_{1}=\frac{\partial}{\partial \mathbf{P}} \cdot \mathcal{A} \cdot \mathbf{P}-\mathbf{P} \cdot \mathcal{A} \cdot \mathbf{P}
$$


where $\mathcal{A}$ is a positive definite symmetric matrix. Then, given any function $\hat{g}(\mathbf{P})$ satisfying $\hat{\mathcal{L}}_{1}^{\dagger} \hat{g}=0, \hat{g}$ has an inverse image of $\hat{\mathcal{L}}_{1}$ of the following form:

$$
\left(\hat{\mathcal{L}}_{1}^{-1} \hat{g}\right)(\mathbf{P})=-\int_{0}^{\infty} \exp \left(\operatorname{Tr} \mathcal{A} t-\frac{1}{2} \mathbf{P} \cdot(\exp (\mathcal{A} t) \cdot \exp (\mathcal{A} t)-\mathcal{I}) \cdot \mathbf{P}\right) \hat{g}(\boldsymbol{\beta}(t)) d t
$$

where

$$
\boldsymbol{\beta}(t)=\exp (\mathcal{A} t) \cdot \mathbf{P}
$$

Note that $\hat{\mathcal{L}}_{1}$ has a one-dimensional kernel (consisting of constant functions), and thus the inverse of $\hat{\mathcal{L}}_{1}$ is not uniquely defined. However, all choices of inverse images under $\hat{\mathcal{L}}_{1}$ differ only by functions of $\mathbf{X}$, which are annihilated in the computation of $\overline{\mathcal{L}}$. Therefore, any choice of inverse image will suffice. Note also that Lemma 2.2 is indeed a generalization of Lemma A.2 in MTV, even though an extra constant parameter appears in the latter; this can always be removed by suitable rescaling of the variables $\mathbf{P}$. We will prove Lemma 2.2 below in section 2.6.

We are now ready to write down the form for the differential operator $\overline{\mathcal{L}}$ describing the effective particle dynamics on time scales $O\left(\mathrm{Ku}_{\mathrm{T}}^{-1}\right)$ in the limit $\mathrm{Ku}_{\mathrm{T}} \rightarrow 0$. Modifying the development in Appendix B of MTV in the manner indicated by Lemma 2.2, we have

$$
\begin{aligned}
\overline{\mathcal{L}} g(\mathbf{X})= & -\mathbf{E}_{O U} \mathcal{L}_{2} \mathcal{L}_{1}^{-1} \mathcal{L}_{2} \mathbf{E}_{O U} g(\mathbf{X}) \\
= & \int_{\mathbb{R}^{N}} d \mathbf{P} \hat{\bar{P}}_{O U}(\mathbf{P}) \hat{\mathcal{L}}_{2} \int_{0}^{\infty} d t\left[\hat{\mathcal{L}}_{2}\left(g(\mathbf{X}) \delta\left(\mathbf{P}^{\prime}\right)\right)\right]_{\mathbf{P}^{\prime}=\boldsymbol{\beta}(\mathbf{P}, t)} \\
& \quad \times \exp \left((\operatorname{Tr} \tilde{\mathcal{R}}) t-\frac{1}{2} \mathbf{P} \cdot(\exp (\tilde{\mathcal{R}} t) \cdot \exp (\tilde{\mathcal{R}} t)-\mathcal{I}) \cdot \mathbf{P}\right),
\end{aligned}
$$

where

$$
\boldsymbol{\beta}(\mathbf{P}, t)=\exp (\tilde{\mathcal{R}} t) \cdot \mathbf{P}
$$

and

$$
\hat{\bar{P}}_{O U}(\mathbf{P})=\exp \left(-\frac{1}{2} \sum_{\boldsymbol{\alpha} \in \mathcal{A}}\left|\mathbf{p}_{\boldsymbol{\alpha}}\right|^{2}\right)
$$

is the invariant measure for the particle velocities, expressed in terms of the Fourier coordinates $\mathbf{P}$. We compute this expression in pieces:

$$
\begin{aligned}
{\left[\hat{\mathcal{L}}_{2}\left(g(\mathbf{X}) \delta\left(\mathbf{P}^{\prime}\right)\right)\right]_{\mathbf{P}^{\prime}=\boldsymbol{\beta}(\mathbf{P}, t)} } & =-i\left[\frac{\partial g}{\partial \mathbf{X}} \cdot \frac{\partial \delta\left(\mathbf{P}^{\prime}\right)}{\partial \mathbf{P}^{\prime}}\right]_{\mathbf{P}^{\prime}=\boldsymbol{\beta}(\mathbf{P}, t)} \\
& =-i(\operatorname{Det}(\exp (\tilde{\mathcal{R}} t)))^{-1} \frac{\partial g}{\partial \mathbf{X}} \cdot \exp (-\tilde{\mathcal{R}} t) \cdot \frac{\partial \delta(\mathbf{P})}{\partial \mathbf{P}} .
\end{aligned}
$$

Thanks to the delta function of $\mathbf{P}$ and the relation $\operatorname{Det}(\exp (\tilde{\mathcal{R}} t))=\exp (\operatorname{Tr} \tilde{\mathcal{R}} t)$, the integral over $t$ can be evaluated to give

$$
\hat{\mathcal{L}}_{1}^{-1} \hat{\mathcal{L}}_{2} \mathbf{E}_{O U} g(\mathbf{X})=i \frac{\partial g}{\partial \mathbf{X}} \cdot \exp (-\tilde{\mathcal{R}} t) \cdot \frac{\partial \delta(\mathbf{P})}{\partial \mathbf{P}}=i \frac{\partial g}{\partial \mathbf{X}} \cdot \tilde{\mathcal{R}}^{-1} \cdot \frac{\partial \delta(\mathbf{P})}{\partial \mathbf{P}},
$$


since $\tilde{\mathcal{R}}$ is a positive definite matrix [6]. Continuing,

$$
\begin{aligned}
\hat{\mathcal{L}}_{2} \hat{\mathcal{L}}_{1}^{-1} \hat{\mathcal{L}}_{2} \mathbf{E}_{O U} g(\mathbf{X})=\operatorname{Tr} \frac{\partial}{\partial \mathbf{X}}\left(\frac{\partial g}{\partial \mathbf{X}} \cdot \tilde{\mathcal{R}}^{-1} \cdot \frac{\partial^{2} \delta(\mathbf{P})}{\partial \mathbf{P} \otimes 2}\right) \\
+\phi \frac{\partial g}{\partial \mathbf{X}} \cdot \tilde{\mathcal{R}}^{-1} \cdot \nabla_{\mathbf{X}} \tilde{\Phi}\left(\frac{\mathbf{X}}{\tilde{\ell}_{f}}\right) \delta(\mathbf{P}) .
\end{aligned}
$$

Finally, integrating against the invariant measure (2.8) for $\mathbf{P}$ and using the symmetry of $\tilde{\mathcal{R}}$, we obtain

$$
\overline{\mathcal{L}} g(\mathbf{X})=\frac{\partial}{\partial \mathbf{X}} \cdot\left(\tilde{\mathcal{R}}^{-1} \cdot \frac{\partial g}{\partial \mathbf{X}}\right)-\phi\left((\tilde{\mathcal{R}})^{-1} \cdot \nabla_{\mathbf{X}} \tilde{\Phi}\left(\frac{\mathbf{X}}{\tilde{\ell}_{f}}\right)\right) \cdot \frac{\partial g}{\partial \mathbf{X}} .
$$

Returning to our more concrete notation, this reduced operator has exactly the (nondimensionalized) Smoluchowski form

$$
\begin{aligned}
\overline{\mathcal{L}} g(\mathbf{X})=\sum_{\boldsymbol{\alpha}, \boldsymbol{\alpha}^{\prime} \in \mathcal{A}} & {\left[\frac{\partial}{\partial \mathbf{X}_{\boldsymbol{\alpha}}} \cdot\left(\left(\tilde{\mathcal{R}}^{-1}\right)_{\boldsymbol{\alpha}, \boldsymbol{\alpha}^{\prime}} \cdot \frac{\partial g}{\partial \mathbf{X}_{\boldsymbol{\alpha}^{\prime}}}\right)\right.} \\
& \left.-\phi\left(\left(\tilde{\mathcal{R}}^{-1}\right)_{\boldsymbol{\alpha}, \boldsymbol{\alpha}^{\prime}} \cdot \nabla_{\boldsymbol{\alpha}^{\prime}} \tilde{\Phi}\left(\frac{\mathbf{X}}{\tilde{\ell}_{f}}\right)\right) \cdot \frac{\partial g}{\partial \mathbf{X}_{\boldsymbol{\alpha}}}\right] .
\end{aligned}
$$

The drift and diffusion coefficients stated in Proposition 2.1 are read off this effective Kolmogorov backward operator in the standard way.

2.5. Generalized mass matrix. The above calculation can be directly generalized to systems of particles with unequal masses or even systems involving nondiagonal mass matrices (as in the case where some coordinates may correspond to rotational degrees of freedom) $[4,5,6]$. The nondimensionalization requires some modification, but this is straightforward for any particular system under consideration. (For example, the reference mass might be chosen as the average mass or mass of the largest particle, etc.) We assume that this nondimensionalization has been done. There will be some other nondimensional parameters characterizing the system (e.g., mass and particle size ratios), but all these will be considered to be held fixed in the low thermal Kubo number limit, and so we do not need to account for them specifically. We restrict attention to the case in which the system variables $\mathbf{X}$ and $\mathbf{V}$ involve only rigid motions of bodies so that the mass matrix is constant.

We start with the nondimensional form of the particle dynamics equations with general nondimensionalized mass matrix $\tilde{\mathcal{M}}$ :

$$
\begin{aligned}
& d \mathbf{X}_{\boldsymbol{\alpha}}(t)=\mathbf{V}_{\boldsymbol{\alpha}}(t) d t \\
& \sum_{\boldsymbol{\alpha}^{\prime} \in \mathcal{A}} \tilde{\mathcal{M}}_{\boldsymbol{\alpha}, \boldsymbol{\alpha}^{\prime}} \cdot d \mathbf{V}_{\boldsymbol{\alpha}^{\prime}}(t)=-\phi \nabla_{\boldsymbol{\alpha}} \tilde{\Phi}\left(\mathbf{X}(t) / \tilde{\ell}_{f}\right) d t \\
& \quad+\sum_{\boldsymbol{\alpha}^{\prime} \in \mathcal{A}}\left[-\mathrm{Ku}_{\mathrm{T}}^{-1} \tilde{\mathcal{R}}_{\boldsymbol{\alpha}, \boldsymbol{\alpha}^{\prime}}(\mathbf{X}(t)) \mathbf{V}_{\boldsymbol{\alpha}^{\prime}}(t) d t+\sqrt{2} \mathrm{Ku}_{\mathrm{T}}^{-1 / 2} \mathcal{S}_{\boldsymbol{\alpha}, \boldsymbol{\alpha}^{\prime}}(\mathbf{X}(t)) d \mathbf{W}_{\boldsymbol{\alpha}^{\prime}}(t)\right]
\end{aligned}
$$

with $\tilde{\mathcal{S}} \tilde{\mathcal{S}}^{\dagger}=\tilde{\mathcal{R}}($ see $[6])$. 
We can transform this system with generalized nondimensional mass matrix to the simplified case considered above (i.e., (2.1)), where the nondimensional mass matrix is the identity matrix, as follows,

$$
\mathbf{V}^{(\tilde{\mathcal{M}})}=\tilde{\mathcal{M}}^{1 / 2} \cdot \mathbf{V}, \quad \mathbf{X}^{(\tilde{\mathcal{M}})}=\tilde{\mathcal{M}}^{1 / 2} \cdot \mathbf{X},
$$

noting that the mass matrix $\tilde{\mathcal{M}}$ is symmetric and positive definite. Multiplying the first equation in (2.9) by $\tilde{\mathcal{M}}^{1 / 2}$ and the second equation in (2.9) by $\tilde{\mathcal{M}}^{-1 / 2}$ and making this change of variables, we find that

$$
\begin{aligned}
& d \mathbf{X}_{\boldsymbol{\alpha}}^{(\tilde{\mathcal{M}})}(t)=\mathbf{V}_{\boldsymbol{\alpha}}^{(\tilde{\mathcal{M}})}(t) d t \\
& d \mathbf{V}_{\boldsymbol{\alpha}}^{(\tilde{\mathcal{M}})}(t)=-\phi \sum_{\boldsymbol{\alpha}^{\prime} \in \mathcal{A}}\left(\tilde{\mathcal{M}}^{-1 / 2}\right)_{\boldsymbol{\alpha}, \boldsymbol{\alpha}^{\prime}} \cdot \nabla_{\boldsymbol{\alpha}^{\prime}} \tilde{\Phi}\left(\mathbf{X}^{(\tilde{\mathcal{M}})}(t) / \tilde{\ell}_{f}\right) d t \\
& +\sum_{\boldsymbol{\alpha}^{\prime} \in \mathcal{A}}\left[-\mathrm{Ku}_{\mathrm{T}}^{-1} \tilde{\mathcal{R}}_{\boldsymbol{\alpha}, \boldsymbol{\alpha}^{\prime}}^{(\tilde{\mathcal{M}})}\left(\mathbf{X}^{(\tilde{\mathcal{M}})}(t)\right) \mathbf{V}_{\boldsymbol{\alpha}^{\prime}}^{(\tilde{\mathcal{M}})}(t) d t+\sqrt{2} \mathrm{Ku}_{\mathrm{T}}^{-1 / 2} \mathcal{S}_{\boldsymbol{\alpha}, \boldsymbol{\alpha}^{\prime}}^{(\mathcal{M})}\left(\mathbf{X}^{(\tilde{\mathcal{M}})}(t)\right) d \mathbf{W}_{\boldsymbol{\alpha}^{\prime}}(t)\right],
\end{aligned}
$$

where

$$
\begin{aligned}
\tilde{\mathcal{R}}^{(\tilde{\mathcal{M}})} & =\tilde{\mathcal{M}}^{-1 / 2} \cdot \tilde{\mathcal{R}} \cdot \tilde{\mathcal{M}}^{-1 / 2}, \\
\mathcal{S}^{(\mathcal{M})} \cdot \mathcal{S}^{(\mathcal{M}) \dagger} & =\tilde{\mathcal{R}}^{(\tilde{\mathcal{M}})} .
\end{aligned}
$$

Consequently, the effective coarse-grained dynamics for a system of particles governed by a general nondimensional mass matrix can be obtained by making the following replacements in Proposition 2.1:

$$
\begin{aligned}
\overline{\mathbf{X}} & \longrightarrow \tilde{\mathcal{M}}^{1 / 2} \cdot \overline{\mathbf{X}} \\
\tilde{\mathcal{R}} & \longrightarrow \tilde{\mathcal{M}}^{-1 / 2} \cdot \tilde{\mathcal{R}} \tilde{\mathcal{M}}^{-1 / 2} \\
\nabla_{\mathbf{X}} \tilde{\Phi}\left(\mathbf{X} / \tilde{\ell}_{f}\right) & \longrightarrow \tilde{\mathcal{M}}^{-1 / 2} \cdot \nabla_{\mathbf{X}} \tilde{\Phi}\left(\mathbf{X} / \tilde{\ell}_{f}\right) .
\end{aligned}
$$

We find in this way that the effective drift and diffusion coefficients for the particle coordinates $\mathbf{X}$ are to be replaced by

$$
\begin{aligned}
\overline{\mathbf{V}}_{\boldsymbol{\alpha}}(\mathbf{X})=\sum_{\boldsymbol{\alpha}^{\prime} \in \mathcal{A}}-\left((\tilde{\mathcal{R}}(\mathbf{X}))^{-1}\right)_{\boldsymbol{\alpha}, \boldsymbol{\alpha}^{\prime}} \cdot \nabla_{\boldsymbol{\alpha}^{\prime}} \tilde{\Phi}\left(\mathbf{X} / \tilde{\ell}_{f}\right) \\
\quad+\sum_{\boldsymbol{\alpha}^{\prime}, \boldsymbol{\alpha}^{\prime \prime} \in \mathcal{A}} \nabla_{\boldsymbol{\alpha}^{\prime}} \cdot\left(\tilde{\mathcal{R}}^{-1}(\mathbf{X})\right)_{\boldsymbol{\alpha}^{\prime}, \boldsymbol{\alpha}^{\prime \prime}}\left(\tilde{\mathcal{M}}^{1 / 2}\right)_{\boldsymbol{\alpha}^{\prime \prime}, \boldsymbol{\alpha}}, \\
\mathcal{S}_{\boldsymbol{\alpha} \boldsymbol{\alpha}^{\prime}}(\mathbf{X})=\sqrt{2}\left(\tilde{\mathcal{R}}^{-1 / 2}(\mathbf{X})\right)_{\boldsymbol{\alpha}, \boldsymbol{\alpha}^{\prime}} .
\end{aligned}
$$

In other words, only the divergence-drift term needs modification by the generalized mass matrix.

2.6. Proof of Lemma 2.2. Following Appendix B of MTV, we write

$$
\hat{\mathcal{L}}_{1}^{-1}=-\int_{0}^{\infty} e^{\hat{\mathcal{L}}_{1} t} d t
$$

Since $\hat{\mathcal{L}}_{1}$ is still a first order differential operator, we can compute $\mathcal{T}(t) \equiv e^{\hat{\mathcal{L}}_{1} t}$ using the method of characteristics. The characteristic equation is

$$
\frac{d \boldsymbol{\Pi}(\boldsymbol{\beta}, t)}{d t}=-\mathcal{A} \Pi(\boldsymbol{\beta}, t), \quad \Pi(\boldsymbol{\beta}, t=0)=\boldsymbol{\beta},
$$


which has solution $\Pi(\boldsymbol{\beta}, t)=\exp (-\mathcal{A} t) \boldsymbol{\beta}$. The transformation to the characteristic coordinate $\boldsymbol{\beta}$ is therefore given by

$$
\boldsymbol{\beta}(\mathbf{P}, t)=\exp (\mathcal{A} t) \mathbf{P} .
$$

Along the characteristics, the evolution operator obeys the ODE

$$
\frac{\partial}{\partial t}\left(\left.\mathcal{T}(t) \hat{g}\right|_{\mathbf{P}=\boldsymbol{\Pi}(\boldsymbol{\beta}, t)}\right)=\left.(\operatorname{Tr} \mathcal{A}-\boldsymbol{\Pi}(\boldsymbol{\beta}, t) \cdot \mathcal{A} \cdot \boldsymbol{\Pi}(\boldsymbol{\beta}, t)) \mathcal{T}(t) \hat{g}\right|_{\mathbf{P}=\boldsymbol{\Pi}(\boldsymbol{\beta}, t)},
$$

which has solution

$$
\left.\mathcal{T}(t) \hat{g}\right|_{\mathbf{P}=\boldsymbol{\Pi}(\boldsymbol{\beta}, t)}=\left.\exp \left(\operatorname{Tr} \mathcal{A} t-\int_{0}^{t} \boldsymbol{\Pi}(\boldsymbol{\beta}, s) \cdot \mathcal{A} \cdot \boldsymbol{\Pi}(\boldsymbol{\beta}, s) d s\right) \hat{g}\right|_{\mathbf{P}=\boldsymbol{\beta}} .
$$

The integral can be evaluated exactly, using the given fact that $\mathcal{A}$ is a symmetric matrix:

$$
\begin{aligned}
\int_{0}^{t} \boldsymbol{\Pi}(\boldsymbol{\beta}, s) \cdot \mathcal{A} \cdot \boldsymbol{\Pi}(\boldsymbol{\beta}, s) d s & =\int_{0}^{t} \boldsymbol{\beta} \cdot \exp (-\mathcal{A} s) \mathcal{A} \exp (-\mathcal{A} s) \cdot \boldsymbol{\beta} \\
& =\frac{1}{2} \boldsymbol{\beta} \cdot(\mathcal{I}-\exp (-\mathcal{A} t) \exp (-\mathcal{A} t)) \cdot \boldsymbol{\beta}
\end{aligned}
$$

Reverting from the characteristic coordinate $\boldsymbol{\beta}$ to the original coordinate $\mathbf{P}$ via (2.11), we have

$$
\mathcal{T}(t) \hat{g}=\exp \left(\operatorname{Tr} \mathcal{A} t-\frac{1}{2} \mathbf{P} \cdot(\exp (\mathcal{A} t) \exp (\mathcal{A} t)-\mathcal{I}) \cdot \mathbf{P}\right) \hat{g}(\boldsymbol{\beta}(\mathbf{P}, t)) .
$$

Upon substitution into (2.10), we obtain the statement in Lemma 2.2.

3. Application of stochastic mode reduction for DPD. The final simulation method which we will treat with the stochastic mode reduction procedure is DPD $[1,7,11,13,23,35,38]$. This simulation scheme has several formal similarities to the PB methods mentioned in section 2 , but differs fundamentally in interpretation. DPD is intended to be used for "complex fluid" systems such as suspensions of polymers, colloids, or other macromolecules. Rather than attempting to resolve the coordinates and configurations of the macromolecules, DPD instead coarse-grains the system in terms of fluid particles which represent some parcel of the fluid suspension. These fluid particles are mesoscopic in size and interact with each other through some force laws that endeavor to capture constitutively the properties of the multiphase mixture. These forces include both conservative and dissipative components. More precisely, under DPD the equations of motion for the fluid particles can be written in the following form $[13,35]$ :

$$
\begin{aligned}
m d \mathbf{V}_{\boldsymbol{\alpha}}(t)= & {\left[-\nabla_{\boldsymbol{\alpha}} \Phi(\mathbf{X}(t))-\sum_{\boldsymbol{\alpha}^{\prime} \neq \boldsymbol{\alpha}} \gamma \omega\left(r_{\boldsymbol{\alpha} \boldsymbol{\alpha}^{\prime}}(t) / \ell_{\gamma}\right)\left(\hat{e}_{\boldsymbol{\alpha} \boldsymbol{\alpha}^{\prime}}(t) \cdot \mathbf{V}_{\boldsymbol{\alpha} \boldsymbol{\alpha}^{\prime}}(t)\right) \hat{e}_{\boldsymbol{\alpha} \boldsymbol{\alpha}^{\prime}}(t)\right] d t } \\
& +\sum_{\boldsymbol{\alpha}^{\prime} \neq \boldsymbol{\alpha}} \sigma \tilde{\omega}\left(r_{\boldsymbol{\alpha} \boldsymbol{\alpha}^{\prime}}(t) / \ell_{\gamma}\right) \hat{e}_{\boldsymbol{\alpha} \boldsymbol{\alpha}^{\prime}}(t) d W_{\boldsymbol{\alpha} \boldsymbol{\alpha}^{\prime}}(t),
\end{aligned}
$$

$$
d \mathbf{X}_{\boldsymbol{\alpha}}(t)=\mathbf{V}_{\boldsymbol{\alpha}}(t) d t
$$


with initial conditions

$$
\mathbf{X}_{\boldsymbol{\alpha}}(t=0)=\mathbf{X}_{0, \boldsymbol{\alpha}}, \quad \mathbf{V}_{\boldsymbol{\alpha}}(t=0)=\mathbf{V}_{0, \boldsymbol{\alpha}}
$$

We use the same notation as in other simulation approaches to describe analogous structures and concepts in the DPD framework. The new notation is the following:

(i) $r_{\boldsymbol{\alpha} \boldsymbol{\alpha}^{\prime}}(t)=\left|\mathbf{X}_{\boldsymbol{\alpha}}(t)-\mathbf{X}_{\boldsymbol{\alpha}^{\prime}}(t)\right|$ is the distance between two DPD particles;

(ii) $\hat{e}_{\boldsymbol{\alpha} \boldsymbol{\alpha}^{\prime}}(t)=\left(\mathbf{X}_{\boldsymbol{\alpha}}(t)-\mathbf{X}_{\boldsymbol{\alpha}^{\prime}}(t)\right) / r_{\boldsymbol{\alpha} \boldsymbol{\alpha}^{\prime}}(t)$ is the unit vector directed from particle $\boldsymbol{\alpha}^{\prime}$ to $\boldsymbol{\alpha}$ cles;

(iii) $\mathbf{V}_{\boldsymbol{\alpha} \boldsymbol{\alpha}^{\prime}}(t)=\mathbf{V}_{\boldsymbol{\alpha}}(t)-\mathbf{V}_{\boldsymbol{\alpha}^{\prime}}(t)$ is the relative velocity between two DPD parti-

(iv) $\gamma$ describes the frictional coupling constant between two DPD particles;

(v) $\sigma$ denotes a normalization factor for the thermal forces;

(vi) $\ell_{\gamma}$ denotes a length scale over which the fluid particles exert thermal and frictional forces on each other (this may loosely be viewed as an effective size of the fluid particles);

(vii) $\omega$ and $\tilde{\omega}$ are dimensionless functions which describe the strength of the frictional and thermal coupling between particles when they are separated by various distances; these functions typically vanish beyond some particle separation distance;

(viii) $\left\{W_{\boldsymbol{\alpha} \boldsymbol{\alpha}^{\prime}}(t)\right\}_{\boldsymbol{\alpha} \neq \boldsymbol{\alpha}^{\prime}}$ is a collection of independent standard real-valued onedimensional Brownian motions (see the discussion near (2.2)) whose differentials describe random thermal force exchanges between particles $\boldsymbol{\alpha}$ and $\boldsymbol{\alpha}^{\prime}$.

The fluctuation-dissipation theorem, or consistency with Gibbs-Boltzmann statistics for a thermal equilibrium state, implies the following relationships between the above parameters:

$$
\gamma=\frac{m \sigma^{2}}{2 k_{B} T}, \quad \omega=\tilde{\omega}^{2} .
$$

Equations (3.1) have a discontinuity when two or more particles coalesce, unless $\omega(r)$ and $\tilde{\omega}(r)$ are chosen to vanish at small $r$. This is physically reasonable, since if the soft fluid particles overlap significantly, the forces they exert on each other should begin to cancel out because of the integration of their interaction over wide angles. The published numerical implementations appear to leave the discontinuity in their simulated equations, which does not matter much because coalescence is a rare event (in three dimensions). For mathematical purposes, it will be convenient to assume that $\omega(r)$ (and therefore also $\tilde{\omega}(r)$ ) vanishes smoothly at $r=0$.

Note that the thermal forces in DPD conserve momentum because the DPD particles alone compose the complex fluid system, in contrast to the rigid particles in the standard $\mathrm{PB}$ simulation method, which can exchange momentum with the fluid medium. Also, note that the friction term in DPD is different from that in the rigid $\mathrm{PB}$ formalism (2.1). We have restricted our attention to isothermal systems so that a common temperature $T$ characterizes all of the DPD fluid particles. The generalizations of DPD to systems with thermal gradients $[1,11]$ involve an extra equation for the dynamics of the internal energy of the fluid particles which does not fit well within the stochastic modeling framework of MTV.

We will obtain here, using the MTV stochastic modeling framework, a simplified formal description of the dynamics of the DPD particles at low thermal Kubo number. This procedure may be viewed as an analogue of the Smoluchowski reduction for the conventional dynamic model of particles in a fluid (section 2), and indeed the results and calculations are rather similar after certain identifications are made. The results 
from the stochastic mode reduction procedure will determine the effective drift and diffusivity of the DPD particles. As these are fictitious particles, it is not so important that these quantities correspond to fundamental physical laws (in contrast to the IB method $[26,25])$.

We must stress that, unlike in the previous sections where the results had a rigorous foundation, the stochastic mode reduction procedure has only a limited formal validity for the DPD system. The reason is that the finite range of the frictional interactions allows the dynamical and uncontrolled appearance of slow modes of particle motion which do not feel frictional damping. The simplified dynamics can be expected to be relevant only for sufficiently dense systems, as we shall explain in section 3.3.4. However, we are not yet able to make this into a rigorous statement.

The coarse-grained dynamics of the DPD particles have previously been characterized in a different way in $[12,34]$ through the calculation of kinetic coefficients such as viscosities of the DPD fluid via Chapman-Enskog expansions. Our framework for calculation provides more detail than these kinetic coefficients in one sense because it incorporates the spatial correlation structure of the DPD fluid particle motion. On the other hand, the stochastic mode elimination framework does not seem well suited to computing quantities such as effective viscosity or pressure of the DPD fluid, since the velocities of the DPD particles, which are needed for these calculations, are fast modes which are eliminated.

\subsection{Nondimensionalization.}

3.1.1. Parameters of externally specified functions. The thermal and frictional coupling has already been expressed in terms of nondimensional functions $\omega$ and $\tilde{\omega}$. We nondimensionalize the potential and initial velocities as we did for PB dynamics,

$$
\Phi(\mathbf{X})=F \ell_{f} \tilde{\Phi}\left(\mathbf{X} / \ell_{f}\right), \quad \mathbf{V}_{0, \boldsymbol{\alpha}}=V_{0} \tilde{\mathbf{V}}_{0, \boldsymbol{\alpha}},
$$

and the initial particle locations as in the IB method,

$$
\mathbf{X}_{0, \boldsymbol{\alpha}}=\ell_{\gamma} \tilde{\mathbf{X}}_{0, \boldsymbol{\alpha}}
$$

this latter nondimensionalization is naturally suggested by the notion that $\ell_{\gamma}$ is a typical fluid particle size and the fluid particles should be space-filling.

3.1.2. Reference units. Here we will use the following:

(i) length scale $\ell_{T}=\ell_{\gamma}$,

(ii) time scale $\tau_{T}=\sqrt{m \ell_{\gamma}^{2} / k_{B} T}$,

(iii) mass scale $m_{T}=m$.

We have chosen these reference units based on thermal (and frictional properties) of the particles, as we did for the other methods. Note that the reference velocity scale,

$$
v_{T} \equiv \frac{\ell_{T}}{\tau_{T}}=\sqrt{\frac{k_{B} T}{m}},
$$

is the order of magnitude which the velocity of the fluid particles would have in thermal equilibrium in the absence of applied forces.

\subsubsection{Nondimensional groups.}

(i) The thermal Kubo number

$$
\mathrm{Ku}_{\mathrm{T}}=\sqrt{\frac{m k_{B} T}{\gamma^{2} \ell_{\gamma}^{2}}} .
$$


If we were to assume that the frictional constant for the DPD particles scales with respect to physical parameters in the same way as rigid particles in viscously overdamped flows $\left(\gamma \propto\left(m / \ell_{\gamma}^{3}\right) \nu \ell_{\gamma}\right)$, then we can also think of $\mathrm{Ku}_{\mathrm{T}}$ as a thermal particle Reynolds number.

(ii) Nondimensionalized measures of the effects of structural forces and initial velocity

$$
\phi=\frac{F \ell_{\gamma}}{k_{B} T}, \quad \Upsilon=\frac{V_{0}}{v_{T}} .
$$

(iii) The length scale ratio

$$
\tilde{\ell}_{f}=\frac{\ell_{f}}{\ell_{\gamma}}
$$

3.1.4. Nondimensionalized DPD equations. Nondimensionalizing with respect to the reference units described in section 3.1.2 and denoting nondimensional variables (but not externally prescribed functions) by the same notation as their dimensional form, we obtain

$$
\begin{aligned}
d \mathbf{X}_{\boldsymbol{\alpha}}(t)= & \mathbf{V}_{\boldsymbol{\alpha}}(t) d t \\
d \mathbf{V}_{\boldsymbol{\alpha}}(t)= & {\left[-\phi \sum_{\boldsymbol{\alpha}^{\prime} \neq \boldsymbol{\alpha}} \nabla_{\boldsymbol{\alpha}} \tilde{\Phi}\left(\mathbf{X}(t) / \tilde{\ell}_{f}\right)\right.} \\
& \left.\quad-\sum_{\boldsymbol{\alpha}^{\prime} \neq \boldsymbol{\alpha}} \mathrm{Ku}_{\mathrm{T}}^{-1} \omega\left(r_{\boldsymbol{\alpha} \boldsymbol{\alpha}^{\prime}}(t)\right)\left(\hat{e}_{\boldsymbol{\alpha} \boldsymbol{\alpha}^{\prime}}(t) \cdot \mathbf{V}_{\boldsymbol{\alpha} \boldsymbol{\alpha}^{\prime}}(t)\right) \hat{e}_{\boldsymbol{\alpha} \boldsymbol{\alpha}^{\prime}}(t)\right] d t \\
& +\sum_{\boldsymbol{\alpha}^{\prime} \neq \boldsymbol{\alpha}} \sqrt{2} \mathrm{Ku}_{\mathrm{T}}^{-1 / 2} \omega^{1 / 2}\left(r_{\boldsymbol{\alpha} \boldsymbol{\alpha}^{\prime}}(t)\right) \hat{e}_{\boldsymbol{\alpha} \boldsymbol{\alpha}^{\prime}}(t) d W_{\boldsymbol{\alpha} \boldsymbol{\alpha}^{\prime}}(t),
\end{aligned}
$$

with initial conditions

$$
\mathbf{X}_{\boldsymbol{\alpha}}(t=0)=\tilde{\mathbf{X}}_{0, \boldsymbol{\alpha}}, \quad \mathbf{V}_{\boldsymbol{\alpha}}(t=0)=\Upsilon \tilde{\mathbf{V}}_{0, \boldsymbol{\alpha}}
$$

3.2. Effective dynamics for DPD particles at low Kubo number. As we shall discuss in section 3.3.4, there is a degeneracy in the DPD equations which does not appear to allow a direct, rigorous application of the theorem by Kurtz [29] to obtain the simplified small Kubo number dynamics. However, it appears that the degeneracy plays only a small role in sufficiently dense systems, and that the formal procedure should be meaningful in these situations. We therefore state the results of stochastic mode reduction on the DPD equations in terms of a formal, nonrigorous proposition.

Proposition 3.1 (DPD at small Kubo number (only formal)). Consider the DPD system (3.1) under the restriction that $\sum_{\boldsymbol{\alpha} \in \mathcal{A}} \nabla_{\boldsymbol{\alpha}} \Phi(\mathbf{X})=0$ and the system is sufficiently dense. Then in the limit $\mathrm{Ku}_{\mathrm{T}} \rightarrow 0$ with all other nondimensional quantities held fixed, the solution for the particle positions $\left\{\mathbf{X}_{\boldsymbol{\alpha}}(t)\right\}_{\boldsymbol{\alpha}}$, obtained from the complete DPD system (3.1) and rescaled in time as

$$
\overline{\mathbf{X}}_{\boldsymbol{\alpha}}(t)=\lim _{\mathrm{Ku}_{\mathrm{T}} \rightarrow 0} \mathbf{X}_{\boldsymbol{\alpha}}\left(t / \mathrm{Ku}_{\mathrm{T}}\right),
$$


can be approximated in some weak sense by the solution of the following simplified stochastic differential system involving only the particle positions $\left\{\overline{\mathbf{X}}_{\boldsymbol{\alpha}}(t)\right\}$ :

$$
\begin{aligned}
d \overline{\mathbf{X}}_{\boldsymbol{\alpha}}(t) & =\overline{\mathbf{V}}_{\boldsymbol{\alpha}}(\overline{\mathbf{X}}(t)) d t+\sum_{\boldsymbol{\alpha}^{\prime} \in \mathcal{A}} \mathcal{S}_{\boldsymbol{\alpha} \boldsymbol{\alpha}^{\prime}}(\overline{\mathbf{X}}(t)) d \mathbf{W}_{\boldsymbol{\alpha}^{\prime}}(t), \\
\overline{\mathbf{X}}_{\boldsymbol{\alpha}}(t=0) & =\tilde{\mathbf{X}}_{0, \boldsymbol{\alpha}}
\end{aligned}
$$

where the stochastic real white noise terms $d \mathbf{W}_{\boldsymbol{\alpha}}(t)$ are defined near (2.2) and are given the Itô interpretation. The explicit expression for the drift term is

$$
\overline{\mathbf{V}}_{\boldsymbol{\alpha}}(\mathbf{X})=-\sum_{\boldsymbol{\alpha}^{\prime} \in \mathcal{A}}\left((\tilde{\mathcal{Q}}(\mathbf{X}))^{-1}\right)_{\boldsymbol{\alpha}, \boldsymbol{\alpha}^{\prime}} \cdot \nabla_{\boldsymbol{\alpha}^{\prime}} \tilde{\Phi}\left(\mathbf{X} / \tilde{\ell}_{f}\right)+\sum_{\boldsymbol{\alpha}^{\prime} \in \mathcal{A}} \nabla_{\boldsymbol{\alpha}^{\prime}}\left(\tilde{\mathcal{Q}}^{-1}(\mathbf{X})\right)_{\boldsymbol{\alpha}^{\prime}, \boldsymbol{\alpha}}
$$

and the matrix coefficients of the stochastic terms are

$$
\mathcal{S}_{\boldsymbol{\alpha} \boldsymbol{\alpha}^{\prime}}(\mathbf{X})=\sqrt{2}\left(\tilde{\mathcal{Q}}^{-1 / 2}(\mathbf{X})\right)_{\boldsymbol{\alpha}, \boldsymbol{\alpha}^{\prime}}
$$

where

$$
\tilde{\mathcal{Q}}_{\boldsymbol{\alpha}, \boldsymbol{\alpha}^{\prime}} \equiv= \begin{cases}\sum_{\boldsymbol{\alpha}^{\prime \prime} \neq \boldsymbol{\alpha}} \omega\left(r_{\boldsymbol{\alpha} \boldsymbol{\alpha}^{\prime \prime}}\right) \hat{e}_{\boldsymbol{\alpha} \boldsymbol{\alpha}^{\prime \prime}} \otimes \hat{e}_{\boldsymbol{\alpha} \boldsymbol{\alpha}^{\prime \prime}} & \text { for } \boldsymbol{\alpha}^{\prime}=\boldsymbol{\alpha} . \\ -\omega\left(r_{\boldsymbol{\alpha} \boldsymbol{\alpha}^{\prime}}\right) \hat{e}_{\boldsymbol{\alpha} \boldsymbol{\alpha}^{\prime}} \otimes \hat{e}_{\boldsymbol{\alpha} \boldsymbol{\alpha}^{\prime}} & \text { for } \boldsymbol{\alpha}^{\prime \prime} \neq \boldsymbol{\alpha}\end{cases}
$$

The inverse of $\tilde{\mathcal{Q}}$ is to be understood as having domain orthogonal to the null-space of $\tilde{\mathcal{Q}}$.

On a formal level, the coarse-grained DPD is quite similar to that of the coarsegrained PB dynamics described in Proposition 2.1; the resistance tensor $\tilde{\mathcal{R}}$ is simply replaced by an effective DPD resistance tensor $\tilde{\mathcal{Q}}$ defined in (3.4). However, the restrictions for the validity of the effective coarse-grained DPD stated in Proposition 3.1 are substantial.

First, the total momentum of the system must be conserved, as for IB dynamics. This rules out consideration of external potentials, which would induce $O(1)$ motion of the center of mass of the DPD particle system over $O(1)$ time scales. This center of mass motion would influence the relative motion of the DPD particles because of the forces applied by the external potential. As with the IB method discussed in [24], we do not know how to generalize our simplified stochastic dynamics to incorporate these changes.

Secondly, the system must be sufficiently dense so that, with large probability, the collection of particles does not (or rarely does) break up into two or more clusters which feel no frictional or thermal coupling with each other. Indeed, in such a configuration, the relative position of the centers of mass of these two clusters would be a degree of freedom that would not feel the strong damping at all and cannot be described within the stochastic mode reduction framework. (It acts as a slow mode, but we cannot identify it a priori!) We discuss this issue below in the context of the actual stochastic mode elimination procedure.

3.3. Stochastic mode reduction for DPD. We now derive Proposition 3.1 following the general prescription from MTV, which we presented in some detail in [24]. We focus here only on those aspects of the calculation that are particular to the DPD equations; the basic notation and formalism are the same as for PB dynamics in section 2.4. We will encounter a technical gap, so the calculations here should only be viewed as formal. 
3.3.1. Kolmogorov backward equation formalism. We identify $\varepsilon=\mathrm{Ku}_{\mathrm{T}}$ as the small parameter and rescale to a longer time $t \rightarrow t / \varepsilon$. We denote the system variables in terms of the collection of particle positions $\mathbf{X}$ and velocities $\mathbf{V}$, as in section 2. Then the Kolmogorov backward equation under this time rescaling can be written as

$$
\begin{aligned}
& -\frac{\partial \rho^{\varepsilon}(s, \mathbf{X}, \mathbf{V} \mid t)}{\partial s}=\varepsilon^{-2} \mathcal{L}_{1} \rho^{\varepsilon}+\varepsilon^{-1} \mathcal{L}_{2} \rho^{\varepsilon}, \\
& \rho^{\varepsilon}(s=t, \mathbf{X}, \mathbf{V} \mid t)=f(\mathbf{X}, \mathbf{V}),
\end{aligned}
$$

with differential operators

$$
\begin{aligned}
& \mathcal{L}_{1}=\sum_{\boldsymbol{\alpha} \neq \boldsymbol{\alpha}^{\prime}} \omega\left(r_{\boldsymbol{\alpha} \boldsymbol{\alpha}^{\prime}}\right)\left[\frac{1}{2}\left(\hat{e}_{\boldsymbol{\alpha} \boldsymbol{\alpha}^{\prime}} \cdot\left(\frac{\partial}{\partial \mathbf{V}_{\boldsymbol{\alpha}}}-\frac{\partial}{\partial \mathbf{V}_{\boldsymbol{\alpha}^{\prime}}}\right)\right)^{2}\right. \\
&\left.-\hat{e}_{\boldsymbol{\alpha} \boldsymbol{\alpha}^{\prime}} \cdot\left(\mathbf{V}_{\boldsymbol{\alpha}}-\mathbf{V}_{\boldsymbol{\alpha}^{\prime}}\right) \hat{e}_{\boldsymbol{\alpha} \boldsymbol{\alpha}^{\prime}} \cdot \frac{\partial}{\partial \mathbf{V}_{\boldsymbol{\alpha}}}\right], \\
& \mathcal{L}_{2}=\sum_{\boldsymbol{\alpha} \in \mathcal{A}}-\phi \nabla_{\boldsymbol{\alpha}} \tilde{\Phi}\left(\frac{\mathbf{X}(t)}{\tilde{\ell}_{f}}\right) \frac{\partial}{\partial \mathbf{V}_{\boldsymbol{\alpha}}}+\mathbf{V}_{\boldsymbol{\alpha}} \cdot \frac{\partial}{\partial \mathbf{X}_{\boldsymbol{\alpha}}} .
\end{aligned}
$$

3.3.2. Asymptotic expansion of solution. We follow the same formalism as in [24], again assuming $f$ depends only on the slow variables $f=f(\mathbf{X})$ to avoid consideration of initial transients. The solvability conditions for the equations in the asymptotic hierarchy are obtained by integrating against the invariant measure $\pi_{O U}$ associated with the operator $\mathcal{L}_{1}$, which here is

$$
\pi_{O U}(\mathbf{V})=\left(\prod_{\boldsymbol{\alpha} \in \mathcal{A}} \frac{1}{2 \pi}\right) \exp \left(-\frac{1}{2} \sum_{\boldsymbol{\alpha} \in \mathcal{A}}\left|\mathbf{V}_{\boldsymbol{\alpha}}\right|^{2}\right) .
$$

The first two equations in the asymptotic hierarchy are solved in the same way as in [24], and the solvability condition resulting from the third equation is again (2.6), so the main task is to compute explicitly

$$
\overline{\mathcal{L}} \equiv-\mathbf{E}_{O U} \mathcal{L}_{2} \mathcal{L}_{1}^{-1} \mathcal{L}_{2} \mathbf{E}_{O U}
$$

3.3.3. Explicit computation of limiting PDE. We again follow the spirit of the calculation in Appendix B of MTV but, as in section 2.4.3, need to make some modifications because $\mathcal{L}_{1}$ is not diagonal in the fast variables $\mathbf{V}$.

We pass again to fast Fourier variables $\mathbf{P}=\left\{\mathbf{p}_{\boldsymbol{\alpha}}\right\}$ defined through

$$
\hat{g}(\mathbf{P})=\int_{\mathbb{R}^{N}} \exp \left[i \sum_{\boldsymbol{\alpha} \in \mathcal{A}} \mathbf{p}_{\boldsymbol{\alpha}} \cdot \mathbf{V}_{\boldsymbol{\alpha}}\right] g(\mathbf{V}) d \mathbf{V} .
$$

The differential operators act on functions of $\mathbf{P}$ and $\mathbf{X}$ as follows:

$$
\begin{aligned}
\hat{\mathcal{L}}_{1} & =\sum_{\boldsymbol{\alpha} \neq \boldsymbol{\alpha}^{\prime}} \omega\left(r_{\boldsymbol{\alpha} \boldsymbol{\alpha}^{\prime}}\right)\left[-\frac{1}{2}\left(\hat{e}_{\boldsymbol{\alpha} \boldsymbol{\alpha}^{\prime}} \cdot\left(\mathbf{p}_{\boldsymbol{\alpha}}-\mathbf{p}_{\boldsymbol{\alpha}^{\prime}}\right)\right)^{2}+\hat{e}_{\boldsymbol{\alpha} \boldsymbol{\alpha}^{\prime}} \cdot\left(\frac{\partial}{\partial \mathbf{p}_{\boldsymbol{\alpha}}}-\frac{\partial}{\partial \mathbf{p}_{\boldsymbol{\alpha}^{\prime}}}\right) \hat{e}_{\boldsymbol{\alpha} \boldsymbol{\alpha}^{\prime}} \cdot \mathbf{p}_{\boldsymbol{\alpha}}\right] \\
& =\sum_{\boldsymbol{\alpha} \neq \boldsymbol{\alpha}^{\prime}} \omega\left(r_{\boldsymbol{\alpha} \boldsymbol{\alpha}^{\prime}}\right)\left[-\frac{1}{2}\left(\hat{e}_{\boldsymbol{\alpha} \boldsymbol{\alpha}^{\prime}} \cdot\left(\mathbf{p}_{\boldsymbol{\alpha}}-\mathbf{p}_{\boldsymbol{\alpha}^{\prime}}\right)\right)^{2}+\hat{e}_{\boldsymbol{\alpha} \boldsymbol{\alpha}^{\prime}} \cdot \frac{\partial}{\partial \mathbf{p}_{\boldsymbol{\alpha}}} \hat{e}_{\boldsymbol{\alpha} \boldsymbol{\alpha}^{\prime}} \cdot\left(\mathbf{p}_{\boldsymbol{\alpha}}-\mathbf{p}_{\boldsymbol{\alpha}^{\prime}}\right)\right], \\
\hat{\mathcal{L}}_{2} & =i \sum_{\boldsymbol{\alpha} \in \mathcal{A}} \phi \mathbf{p}_{\boldsymbol{\alpha}} \cdot \nabla_{\boldsymbol{\alpha}} \tilde{\Phi}\left(\frac{\mathbf{X}(t)}{\tilde{\ell}_{f}}\right)-i \frac{\partial}{\partial \mathbf{p}_{\boldsymbol{\alpha}}} \cdot \frac{\partial}{\partial \mathbf{X}_{\boldsymbol{\alpha}}} .
\end{aligned}
$$


We proceed again as in section 2.4.3 but need to pay special attention to the structure of $\hat{\mathcal{L}}_{1}$. Note that $\hat{\mathcal{L}}_{1}$ has a form satisfying the hypotheses of Lemma 2.2 with the symmetric matrix $\mathcal{A}=\tilde{\mathcal{Q}}$ defined in (3.4), except that we need to take care about positive definiteness. As we discuss in section 3.3.4, we can readily show that $\tilde{\mathcal{Q}}$ is nonnegative definite, but it may possess several nontrivial zero eigenvalues. $\tilde{\mathcal{Q}}$ will always have one zero eigenvalue corresponding to an eigenvector with all entries equal; this simply reflects the fact that the momentum of the center of mass feels no damping. If there are no external forces, this momentum remains constant and can be projected away without difficulty. Of greater concern is the possible presence of additional zero eigenvalues, with eigenvectors depending on the particle configuration. These would imply that certain combinations of the velocities acted as slow rather than fast modes. Since these combinations are configuration-dependent, we cannot simply project them away or reclassify them a priori as slow modes. Zero eigenvalues of $\tilde{\mathcal{Q}}$ beyond the one corresponding to center of mass motion, therefore, would obstruct our ability to obtain simplified dynamics for DPD in the small Kubo number limit. We will refer to particle configurations $\mathbf{X}$ that give rise to multiple zero eigenvalues of $\tilde{\mathcal{Q}}$ as "degenerate configurations." As we shall discuss in section 3.3.4, we cannot exclude the possibility of these degenerate configurations, but the probability that any given realization of the dynamics will pass through a degenerate configuration over some finite time interval should become small as the density of the system increases. (In fact, this probability would appear to remain uniformly small over a fixed interval in the rescaled time $t \rightarrow t / \mathrm{Ku}_{\mathrm{T}}$ as $\mathrm{Ku}_{\mathrm{T}} \rightarrow 0$.) A larger class of realizations may go through degenerate configurations for sufficiently brief periods of time that the coarsegrained dynamics are unaltered. Therefore, we expect that the simplified stochastic dynamical description should apply in some sense to a large fraction of realizations if the density of the system is sufficiently large. To make such a statement have true mathematical sense would require a more proper formulation. Indeed, over any finite time interval, some nonzero fraction of realizations of the simplified dynamics described in Proposition 3.1 will pass through degenerate configurations for which the equations are not well defined; thus it is not appropriate to speak of convergence in law to the formal coarse-grained DPD equations.

We defer further discussion of these important technical matters until section 3.3.4. For now, let us suppose that we are restricting attention to the subset of systems which do not pass through a degenerate configuration, so that $\tilde{\mathcal{Q}}$ has, over the (rescaled) time interval of interest, exactly three zero eigenvalues corresponding to motion of the center of mass. We can project these degrees of freedom away from consideration by working in a frame comoving with the (constant) center of mass velocity, and then, by analogy to (2.7), we compute

$$
\begin{aligned}
\overline{\mathcal{L}} g(\mathbf{X})= & -\mathbf{E}_{O U} \mathcal{L}_{2} \mathcal{L}_{1}^{-1} \mathcal{L}_{2} \mathbf{E}_{O U} g(\mathbf{X}) \\
= & \int_{\mathbb{R}^{N}} d \mathbf{P} \hat{\bar{P}}_{O U}(\mathbf{P}) \hat{\mathcal{L}}_{2} \int_{0}^{\infty} d t\left[\hat{\mathcal{L}}_{2}\left(g(\mathbf{X}) \delta\left(\mathbf{P}^{\prime}\right)\right)\right]_{\mathbf{P}^{\prime}=\boldsymbol{\beta}(\mathbf{P}, t)} \\
& \quad \times \exp \left((\operatorname{Tr} \tilde{\mathcal{Q}}) t-\frac{1}{2} \mathbf{P} \cdot(\exp (\tilde{\mathcal{Q}} t) \cdot \exp (\tilde{\mathcal{Q}} t)-\mathcal{I}) \cdot \mathbf{P}\right),
\end{aligned}
$$

where

$$
\boldsymbol{\beta}(\mathbf{P}, t)=\exp (\tilde{\mathcal{Q}} t) \cdot \mathbf{P}
$$


and

$$
\hat{\bar{P}}_{O U}(\mathbf{P})=\exp \left(-\frac{1}{2} \sum_{\boldsymbol{\alpha} \in \mathcal{A}}\left|\mathbf{p}_{\boldsymbol{\alpha}}\right|^{2}\right) .
$$

Then by making the same kind of calculations as we did for the PB dynamics in section 2.4.3, we obtain

$$
\begin{aligned}
\overline{\mathcal{L}} g(\mathbf{X})=\sum_{\boldsymbol{\alpha}, \boldsymbol{\alpha}^{\prime} \in \mathcal{A}}\left[\frac{\partial}{\partial \mathbf{X}_{\boldsymbol{\alpha}}} \cdot(\right. & \left.\left(\tilde{\mathcal{Q}}^{-1}\right)_{\boldsymbol{\alpha}, \boldsymbol{\alpha}^{\prime}} \cdot \frac{\partial g}{\partial \mathbf{X}_{\boldsymbol{\alpha}^{\prime}}}\right) \\
& \left.-\phi\left(\left(\tilde{\mathcal{Q}}^{-1}\right)_{\boldsymbol{\alpha}, \boldsymbol{\alpha}^{\prime}} \cdot \nabla_{\boldsymbol{\alpha}^{\prime}} \tilde{\Phi}\left(\frac{\mathbf{X}}{\tilde{\ell}_{f}}\right)\right) \cdot \frac{\partial g}{\partial \mathbf{X}_{\boldsymbol{\alpha}}}\right] .
\end{aligned}
$$

This is exactly the Kolmogorov backward differential operator corresponding to the stochastic dynamics recorded in Proposition 3.1.

3.3.4. Positive definiteness of $\tilde{\mathcal{Q}}$. We now discuss in more detail the positive definiteness of $\tilde{\mathcal{Q}}$ in (3.4).

DEFINITION 3.2. Given a frictional coupling function $\omega(r)$, a particle configuration $\mathbf{X}$ will be said to be frictionally connected if for each pair of particles $\boldsymbol{\alpha}$ and $\boldsymbol{\alpha}^{\prime}$ and each pair of unit vectors $\hat{e}, \hat{e}^{\prime} \in \mathbb{R}^{3}$ there exists a finite chain of particles $\left\{\boldsymbol{\alpha}^{(i)}\right\}_{i=1}^{n}$ with $\boldsymbol{\alpha}^{(1)}=\boldsymbol{\alpha}$ and $\boldsymbol{\alpha}^{(n)}=\boldsymbol{\alpha}^{\prime}$ obeying the following conditions:

(i) $\omega\left(\left|\mathbf{X}_{\boldsymbol{\alpha}^{(i+1)}}-\mathbf{X}_{\boldsymbol{\alpha}^{(i)}}\right|\right)>0$ for $1 \leq i \leq n-1$,

(ii) $\left(\mathbf{X}_{\boldsymbol{\alpha}^{(i+1)}}-\mathbf{X}_{\boldsymbol{\alpha}^{(i)}}\right) \cdot\left(\mathbf{X}_{\boldsymbol{\alpha}^{(i)}}-\mathbf{X}_{\boldsymbol{\alpha}^{(i-1)}}\right) \neq 0$ for $2 \leq i \leq n-1$,

(iii) $\hat{e} \cdot\left(\mathbf{X}_{\boldsymbol{\alpha}^{(2)}}-\mathbf{X}_{\boldsymbol{\alpha}^{(1)}}\right) \neq 0$ and $\hat{e}^{\prime} \cdot\left(\mathbf{X}_{\boldsymbol{\alpha}^{(n)}}-\mathbf{X}_{\boldsymbol{\alpha}^{(n-1)}}\right) \neq 0$.

Otherwise, the configuration is said to be frictionally disconnected.

Colloquially, a frictionally connected configuration is one for which any relative motion of any pair of particles induces some sort of frictional damping. This need not be a direct frictional coupling. Imagine, for example, a large but dense cluster of particles with cluster diameter larger than the distance over which frictional coupling acts. Choose two particles at opposite edges of the cluster, and pick arbitrary directions $\hat{e}$ and $\hat{e}^{\prime}$ associated with the respective particles. Suppose that a chain of particles can be found between these two particles such that each pair of successive particles has a nonzero frictional interaction, no two successive pairs have orthogonal separation vectors, and the separation vectors of the pairs at the ends are not orthogonal to $\hat{e}$ and $\hat{e}^{\prime}$. Then any motion of the first particle along $\hat{e}$ and the second particle along $\hat{e}^{\prime}$ will create some frictional damping within the chain, no matter how the other particles are chosen to move along with the two given particles. If no such chain can be found between the two particles, then that implies that they lie in frictionally decoupled subclusters, so that there is an extra degree of freedom (rigid motion of each subcluster) beyond center of mass motion which is not frictionally damped. To understand the nonorthogonality restrictions in the definition of frictional connectedness, note that, for any pair of frictionally interacting particles, the motion of one particle along a line normal to their separation vector incurs frictional damping only at second order in its displacement (and so is vanishing to first order).

Direct calculations establish the following result.

Proposition 3.3. The matrix $\tilde{\mathcal{Q}}$ in (3.4) is always nonnegative definite: $\mathbf{V}$. $\tilde{\mathcal{Q}} \cdot \mathbf{V} \geq 0$ for any vector $\mathbf{V}$. Let $K$ be the null-space of $\tilde{\mathcal{Q}}: \mathbf{V} \in K \leftrightarrow \tilde{\mathcal{Q}} \cdot \mathbf{V}=\mathbf{0}$. This null-space always contains a three-dimensional subspace of vectors characterized 
by $\mathbf{V}_{\boldsymbol{\alpha}}=\mathbf{V}_{\boldsymbol{\alpha}^{\prime}}$ for all $\boldsymbol{\alpha}, \boldsymbol{\alpha}^{\prime} \in \mathcal{A}$. The null-space is strictly larger than this threedimensional subspace whenever the configuration $\mathbf{X}$ is frictionally disconnected.

The general three-dimensional subspace of null vectors corresponds to the center of mass motion, and we can easily eliminate them from our system by a simple linear projection if there is no external potential. The additional vectors in the null-space from frictionally disconnected configurations represent additional undamped modes in the system involving relative motion. For example, if there exists a cluster $\left\{\boldsymbol{\alpha} \in \mathcal{A}_{c}\right\}$ of particles which are not in frictional contact with any particles not in this cluster, then the null-space of $\tilde{\mathcal{Q}}$ contains all vectors $\mathbf{V}$ with the property $\mathbf{V}_{\boldsymbol{\alpha}}=\mathbf{V}_{\boldsymbol{\alpha}^{\prime}}$ for all $\boldsymbol{\alpha}, \boldsymbol{\alpha}^{\prime} \in \mathcal{A}_{c}$, and $\mathbf{V}_{\boldsymbol{\alpha}}=0$ for all $\boldsymbol{\alpha} \notin \mathcal{A}_{c}$.

CONJECTURE 3.4. For frictionally connected configurations $\mathbf{X}$, the null-space of the matrix $\tilde{\mathcal{Q}}$ is precisely the three-dimensional space described in Proposition 3.3. There are no additional null eigenvectors.

The reason for the conjecture is that, from a physical standpoint, the system has no undamped relative degrees of freedom other than center of mass motion. We have not been able to provide a mathematical proof, however. The matrix $\tilde{\mathcal{Q}}$ has the form of an infinitesimal generator of a continuous time Markov chain [42], except that the off-diagonal components consist of nonnegative dyadic matrices rather than nonnegative scalars. The usual arguments for characterizing the null-space of an infinitesimal generator, however, suffered algebraic defects when we tried to apply them to the case at hand.

If we grant the conjecture, then a sufficiently dense system should remain frictionally connected with high probability, and one could imagine applying Kurtz's theorem [29] only on such realizations. A proper technical framework, however, would need to be constructed to make such a statement mathematically meaningful, and this is beyond the scope of the present work.

4. Conclusion. The MTV stochastic mode reduction procedure provides a way to recover rigorously the Smoluchowski equation for PB models at low thermal Kubo number. In contrast, the governing equations for DPD have an algebraic structure amenable to the MTV procedure, but they incur analytical obstacles due to the finite range of the frictional interaction. This feature introduces a mobile degeneracy which prevents the a priori identification of the fast and slow modes in the system; slow modes can, rather, emerge dynamically. It would be interesting to formulate a mathematically sound way to obtain and describe the simplified dynamics of such a system, particularly when the degeneracies occur rarely.

Acknowledgments. The authors thank Eric Vanden-Eijnden for helpful discussions and suggestions, and the referees for constructive comments which improved the presentation.

\section{REFERENCES}

[1] J. B. Avalos And A. D. Mackie, Dissipative particle dynamics with energy conservation, Europhys. Lett., 40 (1997), pp. 141-146.

[2] D. A. BEARD AND T. SChlick, Inertial stochastic dynamics. II. Influence of inertia on slow kinetic processes of supercoiled DNA, J. Comput. Phys., 112 (2000), pp. 7323-7338.

[3] L. Bocquet, High friction limit of the Kramers equation: The multiple time-scale approach, Amer. J. Phys., 65 (1997), pp. 140-144.

[4] G. Bossis and J. F. Brady, Dynamic simulation of sheared suspensions. I. General method, J. Chem. Phys., 80 (1984), pp. 5141-5154.

[5] J. F. Brady, Brownian motion, hydrodynamics, and the osmotic pressure, J. Chem. Phys., 98 (1993), pp. 3335-3341. 
[6] J. F. Brady and G. Bossis, Stokesian dynamics, in Annu. Rev. Fluid Mech. 20, Annual Reviews, Palo Alto, CA, 1988, pp. 111-157.

[7] W. K. Den Otter and J. H. R. Clarke, A new algorithm for dissipative particle dynamics, Europhys. Lett., 53 (2001), pp. 426-431.

[8] J. M. Deutch And I. Oppenheim, The concept of Brownian motion in modern statistical mechanics, in Brownian Motion, Faraday Discussions of the Chemical Society 83, The Royal Society of Chemistry, London, 1987, pp. 1-20.

[9] B. Dünweg, G. S. Grest, And K. Kremer, Molecular dynamics simulations of polymer systems, in Numerical Methods for Polymeric Systems (Minneapolis, MN, 1996), Springer, New York, 1998, pp. 159-195.

[10] D. L. Ermak and J. A. McCammon, Brownian dynamics with hydrodynamic interactions, J. Chem. Phys., 69 (1978), pp. 1352-1360.

[11] P. Español, Dissipative particle dynamics with energy conservation, Europhys. Lett., 40 (1997), pp. 631-636.

[12] P. Español, Fluid particle model, Phys. Rev. E, 57 (1998), pp. 2930-2948.

[13] P. Español and P. Warren, Statistical mechanics of dissipative particle dynamics, Europhys. Lett., 30 (1995), pp. 191-196.

[14] S. N. Ethier And T. G. Kurtz, Markov Processes: Characterization and Convergence, John Wiley \& Sons, New York, 1986.

[15] The Faraday Division, Concentrated Colloidal Dispersions, Faraday Discussions of the Chemical Society 76, The Royal Society of Chemistry, London, 1983.

[16] The Faraday Division, Brownian Motion, Faraday Discussions of the Chemical Society 83, The Royal Society of Chemistry, London, 1987.

[17] M. Fixman, Brownian dynamics of chain polymers, in Brownian Motion, Faraday Discussions of the Chemical Society 83, The Royal Society of Chemistry, London, 1987, pp. 1-20.

[18] C. W. Gardiner, Handbook of Stochastic Methods, Springer Ser. Synergetics 13, 2nd ed., Springer-Verlag, Berlin, 1985.

[19] P. S. Grassia, E. J. Hinch, And L. C. Nitsche, Computer simulations of Brownian motion of complex systems, J. Fluid Mech., 282 (1995), pp. 373-403.

[20] J. Happel And H. Brenner, Low Reynolds Number Hydrodynamics with Special Applications to Particulate Media, Prentice-Hall, Englewood Cliffs, NJ, 1965.

[21] E. H. Hauge And A. Martin-LÖF, Fluctuating hydrodynamics and Brownian motion, J. Statist. Phys., 7 (1973), pp. 259-281.

[22] W. Hess And R. Klein, Dynamical properties of colloidal systems. 1. Derivation of stochastic transport equations, Phys. A, 94 (1978), pp. 71-90.

[23] P. J. Hoogerbrugge and J. M. V. A. Koelman, Simulating microscopic hydrodynamic phenomena with dissipative particle dynamics, Europhys. Lett., 19 (1992), pp. 155-160.

[24] P. R. Kramer And A. J. MajDA, Stochastic mode reduction for the immersed boundary method, SIAM J. Appl. Math., 64 (2003), pp. 369-400.

[25] P. R. Kramer and C. S. Peskin, An Extension of the Immersed Boundary Method Including Thermal Fluctuations, manuscript, 2003.

[26] P. R. Kramer and C. S. Peskin, Incorporating thermal fluctuations into the immersed boundary method, in Proceedings of the Second MIT Conference on Computational Fluid and Solid Mechanics, Cambridge, MA, 2003, Elsevier Science, Oxford, UK, 2003, pp. 17551758.

[27] H. A. Kramers, Brownian motion in a field of force and the diffusion model of chemical reactions, Physica, 7 (1940), pp. 284-304.

[28] M. Kröger, A. Alba-Pérez, M. Laso, And H. C. Öttinger, Variance reduced Brownian simulation of a bead-spring chain under steady shear flow considering hydrodynamic interaction effects, J. Chem. Phys., (2000), pp. 4767-4773.

[29] T. G. KuRTz, A limit theorem for perturbed operator semigroups with applications to random evolutions, J. Funct. Anal., 12 (1973), pp. 55-67.

[30] H. A. Lorentz, Lessen über Theoretische Natuurkunde: Kinetische Problemen, Vol. 5, E. J. Brill, 1921.

[31] A. Majda, I. Timofeyev, and E. Vanden-Eijnden, A priori tests of a stochastic mode reduction strategy, Phys. D, 170 (2002), pp. 206-252.

[32] A. J. Majda, I. Timofeyev, and E. Vanden-Eijnden, Models for stochastic climate prediction, Proc. Natl. Acad. Sci. USA, 96 (1999), pp. 14687-14691.

[33] A. J. Majda, I. Timofeyev, and E. Vanden-Eijnden, A mathematical framework for stochastic climate models, Comm. Pure Appl. Math., 54 (2001), pp. 891-974.

[34] C. A. Marsh, G. Backx, AND M. H. ERnst, Fokker-Planck-Boltzmann equation for dissipative particle dynamics, Europhys. Lett., (1997), pp. 411-415.

[35] C. A. Marsh and J. M. Yeomans, Dissipative particle dynamics: The equilibrium for finite 
time steps, Europhys. Lett., 37 (1997), pp. 511-516.

[36] A. J. MASTERS, Time-scale separations and the validity of the Smoluchowski, Fokker-Planck and Langevin equations as applied to concentrated particle suspensions, Molec. Phys., 57 (1986), pp. 303-317.

[37] T. J. Murphy and J. L. Aguirre, Brownian motion of $N$ interacting particles. I. Extension of the Einstein diffusion relation to the $N$-particle case, J. Chem. Phys., 57 (1972), pp. 2098-2104.

[38] I. Pagonabarraga, M. H. J. Hagen, and D. Frenkel, Self-consistent dissipative particle dynamics algorithm, Europhys. Lett., 42 (1998), pp. 377-382.

[39] G. C. Papanicolaou, Some probabilistic problems and methods in singular perturbations, Rocky Mountain J. Math., 6 (1976), pp. 653-674.

[40] C. S. Peskin, The immersed boundary method, Acta Numer., 11 (2002), pp. 479-517.

[41] P. N. Pusey AND R. J. A. Tough, Hydrodynamic interactions and diffusion in concentrated particle suspensions, in Concentrated Colloidal Suspensions, Faraday Discussions of the Chemical Society 76, The Royal Society of Chemistry, London, 1983, pp. 123-136.

[42] S. Resnick, Adventures in Stochastic Processes, Birkhäuser-Boston, Boston, MA, 1992.

[43] J. Rotne and S. Prager, Variational treatment of hydrodynamic interaction in polymers, J. Chem. Phys., 50 (1969), pp. 4831-4837.

[44] J.-N. Roux, Brownian particles at different times scales: A new derivation of the Smoluchowski equation, Phys. A, 188 (1992), pp. 526-552.

[45] J. Schröter, The complete Chapman-Enskog procedure for the Fokker-Planck equation, Arch. Ration. Mech. Anal., 66 (1977), pp. 183-199.

[46] A. Sierou And J. F. Brady, Accelerated Stokesian dynamics simulations, J. Fluid Mech., 448 (2001), pp. 115-146.

[47] U. M. TITULAER, Corrections to the Smoluchowski equation in the presence of hydrodynamic interactions, Phys. A, 100 (1980), pp. 251-265.

[48] M. TokUYama And I. Oppenheim, Statistical-mechanical theory of Brownian motionTranslational motion in an equilibrium fluid, Phys. A, 94 (1978), pp. 501-520.

[49] N. G. Van Kampen and I. Oppenheim, Brownian motion as a problem of eliminating fast variables, Phys. A, 138 (1986), pp. 231-248.

[50] G. Wilemski, On the derivation of Smoluchowski equations with corrections in the classical theory of Brownian motion, J. Statist. Phys., 14 (1976), pp. 153-169. 